\title{
FLRT2 and FLRT3 Cooperate in Maintaining the Tangential Migratory Streams of Cortical Interneurons during Development
}

\author{
Catherine Fleitas, ${ }^{1 *}$ Pau Marfull-Oromí, ${ }^{1 *}$ Disha Chauhan, ${ }^{1}$ Daniel del Toro, ${ }^{2}$ Blanca Peguera, ${ }^{1,3}$ \\ Bahira Zammou, ${ }^{1}$ Daniel Rocandio, ${ }^{1}$ Rüdiger Klein, ${ }^{2}$ Carme Espinet, ${ }^{1}$ and ${ }^{\circledR}$ Joaquim Egea ${ }^{1,4}$ \\ ${ }^{1}$ Lleida Biomedical Research Institute, University of Lleida, Lleida 25198, Spain, ${ }^{2}$ Max Planck Institute of Neurobiology, 82152 Martinsried, \\ Germany, ${ }^{3}$ Institute of Cell Biology and Neuroscience and Buchmann Institute for Molecular Life Sciences, University of Frankfurt, D-60438 \\ Frankfurt am Main, Germany, and ${ }^{4}$ Serra Hunter Associate Professor, Government of Catalonia, 08007, Spain
}

Neuron migration is a hallmark of nervous system development that allows gathering of neurons from different origins for assembling of functional neuronal circuits. Cortical inhibitory interneurons arise in the ventral telencephalon and migrate tangentially forming three transient migratory streams in the cortex before reaching the final laminar destination. Although migration defects lead to the disruption of inhibitory circuits and are linked to aspects of psychiatric disorders such as autism and schizophrenia, the molecular mechanisms controlling cortical interneuron development and final layer positioning are incompletely understood. Here, we show that mouse embryos with a double deletion of FLRT2 and FLRT3 genes encoding cell adhesion molecules exhibit an abnormal distribution of interneurons within the streams during development, which in turn, affect the layering of somatostatin + interneurons postnatally. Mechanistically, FLRT2 and FLRT3 proteins act in a noncell-autonomous manner, possibly through a repulsive mechanism. In support of such a conclusion, double knockouts deficient in the repulsive receptors for FLRTs, Unc5B and Unc5D, also display interneuron defects during development, similar to the FLRT2/FLRT3 mutants. Moreover, FLRT proteins are chemorepellent ligands for developing interneurons in vitro, an effect that is in part dependent on FLRT-Unc5 interaction. Together, we propose that FLRTs act through Unc5 receptors to control cortical interneuron distribution in a mechanism that involves cell repulsion.

Key words: cortex; development; FLRT; interneuron; migration; Unc5

Significance Statement

Disruption of inhibitory cortical circuits is responsible for some aspects of psychiatric disorders such as schizophrenia or autism. These defects include interneuron migration during development. A crucial step during this process is the formation of three transient migratory streams within the developing cortex that determine the timing of interneuron final positioning and the formation of functional cortical circuits in the adult. We report that FLRT proteins are required for the proper distribution of interneurons within the cortical migratory streams and for the final laminar allocation in the postnatal cortex. These results expand the multifunctional role of FLRTs during nervous system development in addition to the role of FLRTs in axon guidance and the migration of excitatory cortical neurons.

Received Feb. 18, 2020; revised June 29, 2021; accepted July 6, 2021.

Author contributions: C.F., P.M.-O., D.C., D.d.T., B.P., B.Z., and D.R. designed research; C.F., P.M.-O., D.C., D.d.T., B.P., B.Z., and D.R. performed research; J.E., C.F., P.M.-O., D.C., D.d.T., B.P., B.Z., D.R., R.K., and C.E. analyzed data; J.E., R.K., and C.E. wrote the paper.

This work was supported by grants from the Spanish Ministry of Science and Innovation (BFU2010-1805, BFU201348563-P, and PGC2018-101910-B-100 to J.E. and BES-2014-067618 to P.M.-0.), FP7-PEOPLE-2011-CIG (PCIG9-GA-2011293980 to J.E.), the Max-Planck Society (R.K.), and the Jade Plus Fellowship Program 2011-2014 (C.F.). We thank Tristan Rodríguez (Sox1-Cre line) and Anne Eichmann (Unc5B line) for the transgenic mice; Michèle Studer for the vasointestinal peptide probe; Eve Seuntjens and Veronique van den Berghe for scientific discussion; Inmaculada Segura for reading the manuscript; Serafí Cambray, Alex Espinós, Ma José Menal, Inma Montoliu, Montse Ortega, Noel Pérez,
Sònia Rius, Marc Tarrés, and the University of Lleida Scientific and Technical Services for technical assistance, and the University of Lleida animal house staff facility for animal care.

D. del Toro's present address: IDIBAPS (August Pi i Sunyer Biomedical Research Institute), Network Center for Biomedical Research in Neurodegenerative Diseases, University of Barcelona, Barcelona, Spain.

*C.F. and P.M.-O. contributed equally to this work.

The authors declare no competing financial interests.

Correspondence should be addressed to Joaquim Egea at joaquim.egea@udl.cat.

https://doi.org/10.1523/JNEUROSCI.0380-20.2021

Copyright $\odot 2021$ the authors 


\section{Introduction}

The cerebral cortex is the main brain region processing higher information including cognition, language, and abstract thinking (Kandel et al., 2000). Cortical neuronal circuits are formed by excitatory, pyramidal-shaped neurons and by more heterogeneous inhibitory GABAergic interneurons (Tremblay et al., 2016). Disruption of the inhibitory circuits underlies some clinical features of several psychiatric disorders including autism or schizophrenia (Marín, 2012). Interestingly, these defects may already occur during development (Ross and Walsh, 2001).

Cortical interneurons arise from progenitors in the subventricular (SVZ) and the ventricular (VZ) zones, mainly in the medial ganglionic eminence (MGE) of the ventral telencephalon (Evsyukova et al., 2013). Within the developing cortex, interneurons follow two main tangential migratory streams located in the marginal zone (MZ) and the boundary between the lower intermediate zone (IZ) and the SVZ, avoiding the cortical plate (CP; Tanaka and Nakajima, 2012). However, a smaller fraction of interneurons migrate through the subplate (SP; HernándezMiranda et al., 2010). Later in development, interneurons switch to a radial-orientated migration and enter the $\mathrm{CP}$ to reach their final laminar destination and integrate in neuronal circuits with excitatory neurons (Nadarajah et al., 2002; Ang et al., 2003; Tanaka et al., 2003; Faux et al., 2012; Bartolini et al., 2017). Tangential migration and the timing of CP invasion are therefore crucial events for the proper formation of functional cortical interneuron circuitry.

Interneuron migration is controlled by intrinsic genetic programs, cytoskeleton dynamics, extrinsic signals including motogenic and neurotrophic factors and chemoattractive or chemorepulsive cues (Peyre et al., 2015). In the cortex, the potent chemoattractive chemokine SDF1 (C-X-C motif chemokine 12 or Cxcl12) and its receptors $\mathrm{Cxcr} 4$ and $\mathrm{Cxcr} 7$ are necessary and sufficient to organize the cortical migratory streams (Tiveron et al., 2006; Li et al., 2008; López-Bendito et al., 2008; Sánchez-Alcañiz et al., 2011; Wang et al., 2011; Zarbalis et al., 2012). In contrast, transcriptomic analysis indicates that specification of interneuron diversity is established early during development and that interneurons in each stream display a specific genetic expression profile (Antypa et al., 2011; Mi et al., 2018). Interestingly, early specification seems to determine the choice of the migratory cortical route, indicating the presence of specific signals involved in sorting interneurons between the different migratory streams (Lim et al., 2018). However, the identity of these stream-specific signals is largely unknown.

The fibronectin leucine-rich transmembrane (FLRT1-3) proteins family plays important functions during excitatory neuron development including neuron migration, axon guidance, or synapse formation (Yamagishi et al., 2011; O'Sullivan et al., 2012; Leyva-Díaz et al., 2014; Seiradake et al., 2014; del Toro et al., 2017; 2020). Mechanistically, FLRTs display some redundant and unique multifunctional properties acting as chemorepellents by binding to the Netrin receptor Unc5D and regulating radial migration of SVZ progenitors (Yamagishi et al., 2011; Seiradake et al., 2014), as well as homophilic adhesion molecules regulating the tangential dispersion of migrating neuron progenitors in the developing cortex (Seiradake et al., 2014; del Toro et al., 2017). Moreover, recent evidence suggests that radial neuron migration can be regulated by a ternary complex involving FLRT proteins and the synaptogenic complex teneurin-latrophilin (del Toro et al., 2020). Considering these effects, it is conceivable that FLRTs may regulate the development of other cortical neuron populations such as interneurons.

Here, we studied the role of FLRTs in tangential migration of cortical interneurons. Double FLRT2;FLRT3 genetic ablation resulted in a disorganized distribution of cortical interneurons during brain development, affecting mainly those allocated in the SP stream. Postnatal mutants displayed abnormal positioning of somatostatin $(\mathrm{SST}+)$ interneurons in the cortex, whereas the distribution of parvalbumin $(\mathrm{PV}+)$ or calretinin $(\mathrm{CR}+)$ interneurons remained unaltered. Conditional deletion experiments demonstrated that FLRT2 and FLRT3 displayed a noncell-autonomous effect in vivo. Finally, we provide evidence that FLRTs act as chemorepellent signals to MGE-derived interneurons in vitro through at least Unc5 receptors. In summary, we have revealed that FLRTs are important for interneuron development and final cortical allocation in vivo.

\section{Materials and Methods}

Mice. Animal care was in accordance with the Guidelines of the University of Lleida for Animal Experimentation in accordance with Catalan, Spanish, and European Union regulations (Decret 214/1997, Real Decreto 53/2013, and Directive 63/2010). Animals were housed in the animal house of the University of Lleida with 12:12 h light/dark cycle and food/water available ad libitum. The mouse lines Emx1-Cre [catalog \#4834018, Mouse Genome Informatics (MGI); RRID:MGI:4834018], Sox1-Cre [catalog \#RBRC05065, International Mouse Strain Resource (IMRS); RRID:IMSR_RBRC05065], Nestin-Cre (catalog \#JAX:019103, IMSR; RRID:IMSR_JAX:019103), FLRT2 ${ }^{\text {null }}$ (catalog \#5495541, MGI: RRID:MGI:5495541), FLRT3 ${ }^{\text {null }}$ (catalog \#3821880, MGI: RRID: MGI:3821880), FLRT3 ${ }^{l x}$ (catalog \#5695860, MGI; RRID:MGI:5695860), $U_{n c 5 D^{\text {null }}}$ (catalog \#5495543, MGI; RRID:MGI:5495543), Unc5B ${ }^{\text {null }}$ (catalog \#3760992, MGI; RRID:MGI:3760992) and $U n c 5 B^{l x}$ (catalog \#3654307, MGI; RRID:MGI:3654307) were previously described (Lu et al., 2004; Wilson et al., 2006; Takashima et al., 2007; Egea et al., 2008; Yamagishi et al., 2011; Paixão et al., 2013). The Sox1-Cre line was included in this analysis because FLRT2 and Nestin-Cre loci do not segregate effectively as they are closely located in the same chromosome. An embryonic stem cell clone $\left(F L R T 2^{t m l a(E U C O M M) W t s i}\right)$ obtained from the European Conditional Mouse Mutagenesis Program was used to generate $F L R T 2^{l x}$ mice by blastocyst injection (Transgenic Service, Max Planck Institute of Neurobiology). The B6.129 × 1-Gt(ROSA)26Sortm1(EYFP)Cos/J (stock \#006148, IMSR; RRID: IMSR_JAX:006148) mouse line (R26R-EYFP) bearing a loxP-flanked STOP sequence followed by the enhanced yellow fluorescent protein gene (EYFP) inserted into the Gt(ROSA)26Sor locus and the Dlx5/6-Cre ( Tg(dlx5a-cre) $1 M e k k / J$ (stock \#008199; RRID:IMSR_JAX:008199) were obtained from The Jackson Laboratory. Single mutants (FLRT2 ${ }^{\text {null/lx }}$;Sox1Cre+; FLRT3 ${ }^{\text {null/lx }}$;Sox1-Cre+; FLRT3 ${ }^{\text {null/l/x}}$;Nestin-Cre+) were obtained by crossing males FLRT2 $^{+/ \text {null }}$;Sox1-Cre ${ }^{+}$, FLRT3 $^{+/ \text {null }}$;Sox $1-C r e^{+}$, or FLRT3 $^{+/ \text {null }} ;$ Nestin-Cre $^{+}$with females FLRT2 $2^{l x / l x}$ or FLRT3 ${ }^{l x / l x}$, respectively. Double mutants $\left(F L R T 2^{\text {null/lx }} ; F L R T 3^{\text {null/lx }} ;\right.$ Sox 1-Cre+; FLRT2 $2^{\text {null/lx }} ;$ FLRT3 $3^{\text {null/ll }} ;$ Emx1-Cre+) were obtained by crossing

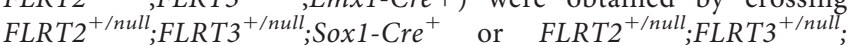
Emx1-Cre ${ }^{+}$males with FLRT2 ${ }^{l x / l x} ; F L R T 3^{l x / l x}$ females, respectively. Littermates with other genotypes were pooled and used as controls. Transgenic mice were backcrossed in C57/bl6 background. For embryo dissection, the day of positive vaginal plug was considered as embryonic day 0.5 . The date of birth was considered as postnatal (P) day 0.

In situ hybridization. Embryonic brains were dissected and fixed overnight in $4 \%$ paraformaldehyde (PFA)/PBS, cryoprotected in $30 \%$ sucrose in PBS, embedded in cryoprotective Tissue-Tek O.C.T. Compound (Sakura) and stored at $-80^{\circ} \mathrm{C}$. Serial $16 \mu \mathrm{m}$ coronal sections were made in cryostat (Leica CM3000), collected in Superfrost Plus slides (Thermo Fisher Scientific) and hybridized with digoxigenin-labeled cDNA FLRT2, FLRT3, or vasointestinal peptide (VIP) probes as previously described (Egea et al., 2008; Lodato et al., 2011).

Immunofluorescence, fluorescent microscopy, and quantification of staining. Embryonic brains were fixed for $4 \mathrm{~h}$ in $4 \%$ PFA/PBS. P21 mice were anesthetized with Rompun (xylacine, $0.01 \mathrm{mg} / \mathrm{g}$; Bayer)/Imalgene (ketamine, $0.1 \mathrm{mg} / \mathrm{g}$; Merial), perfused transcardially with PBS followed by $4 \% \mathrm{PFA} / \mathrm{PBS}$, and the brain was dissected and fixed overnight in $4 \%$ 
PFA/PBS. After fixation, brains were processed as for in situ hybridization (see above). The slides containing the sections ( $16 \mu \mathrm{m}$ for embryonic brains or $30 \mu \mathrm{m}$ for adult brains) were washed with PBS, permeabilized, and blocked with $5 \%$ donkey serum in $0.1 \%$ Triton X-100 in PBS for $1 \mathrm{~h}$ at room temperature. Subsequently, the slides were incubated with primary antibodies diluted in blocking buffer for $12-24 \mathrm{~h}$ at $4^{\circ} \mathrm{C}$. After washing, the appropriate fluorescent secondary antibodies, diluted in blocking buffer containing DAPI, were used $(2 \mathrm{~h}$ at room temperature). Samples were mounted with antifading mounting medium Fluoromount-G (SouthernBiotech) and visualized with an upright fluorescence microscope (Olympus BX51) or with a confocal laser scanning biological microscope (FLUOVIEW FV1000, Olympus). Brightness and contrast were linearly adjusted with Adobe Photoshop. Callbindin $(\mathrm{CB})+$ cells in the MZ were quantified using Image J and CellProfiler (Lamprecht et al., 2007). Anatomical regions of the cortex were determined using DAPI counterstaining and specific layering markers. Rostral, intermediate, and caudal positions in the embryonic brain correspond to sections 6-9, 10-11, and 13-14 of gestational day 14 defined in Schambra (2009).

Antibodies for immunofluorescence. Antibodies include the following: Goat anti-FLRT2 ECD (1:100; catalog \#AF2877, R\&D Systems; RRID:AB_2106600), goat anti-FLRT3 ECD (1:100; catalog \#AF2795, R\&D Systems; RRID:AB_2106855), rabbit anti-Calbindin (1:100; catalog \#CB-38, Swant; RRID:AB_10000340), rabbit anti-Calretinin (1:100; catalog \#CR-7697, Swant; RRID:AB_2619710), rabbit anti-Tbr1 (1:100; catalog \#ab31940, Abcam; RRID:AB_2200219), rat anti-Ctip2 (1:100; catalog \#ab18465, Abcam; RRID:AB_2064130), mouse anti-Satb2 (1:100; catalog \#ab51502, Abcam; RRID:AB_882455), rabbit anti-Parvalbumin (1:100; catalog \#PV-25, Swant; RRID:AB_10000344), and rat anti-Somatostatin (1:100; catalog \#MAB354, Millipore; RRID:AB_2255365). Cyanine or alexa-conjugated secondary antibodies were raised in donkey and purified for multilabelling purposes (1:300; Jackson ImmunoResearch).

Interneuron migration and stimulation in MGE cultures. Pieces of MGE from E14.5 embryos were embedded in a drop of Matrigel (Becton Dickinson) and cultured in p35 plates with neurobasal medium supplemented with B27 (Invitrogen), L-glutamine (Invitrogen) and antibiotics (penicillin and streptomycin) at $37^{\circ} \mathrm{C}$ in a $5 \% \mathrm{CO}_{2}$-humidified incubator. Images were acquired at 24 and $48 \mathrm{~h}$ using an Olympus BX51 inverted microscope. Migration was quantified as the maximum distance achieved by interneurons away from the center of the explant and expressed as fold change in relation to the radius of the explant. Measurements were taken randomly every $20^{\circ}$ along the perimeter of the explant (see Fig. 7A). Stimulation of MGE explants with the recombinant proteins $(2 \mu \mathrm{g} / \mathrm{ml}$ final concentration) was performed for $30 \mathrm{~min}$ at $37^{\circ} \mathrm{C}$ in the cell culture incubator. Prior to stimulation, the recombinant proteins $\mathrm{Fc}$ (Jackson ImmunoResearch), FLRT2 ${ }^{\mathrm{ECD}}-\mathrm{Fc}$ or FLRT $3^{\mathrm{ECD}}$-Fc (purified as described in Seiradake et al., 2014) were preclustered for $60 \mathrm{~min}$ at room temperature with Alexa Fluor 594 goat antihuman IgG (Life Technologies) in a 5:1 ratio. After stimulation, cultures were washed with PBS, fixed, permeabilized, and processed for immunofluorescence as above.

Biochemistry and Western blot. Lysates from embryonic brains were obtained as previously described (Egea et al., 2005). Protein quantification was determined by detergent compatible Protein Assay (Bio-Rad). Glycoproteins were pulled down from $400 \mu \mathrm{g}$ of protein lysate using wheat germ agglutinin (lectin) agarose (Sigma). Proteins were separated by SDSPAGE (Bio-Rad) and transferred to PVDF membranes (Millipore). Western blots were performed using primary antibodies against FLRT2 (anti-FLRT2 ECD, 1:1000; catalog \#AF2877, R\&D Systems; RRID:AB_ 2106600) and FLRT3 (goat anti-FLRT3 ECD, 1:1000; catalog \#AF2795, R\&D Systems; RRID:AB_2106855) or Tubulin (anti- $\alpha$-Tubulin, catalog \#T5168, Sigma-Aldrich; RRID:AB_477579) and EphA4 (anti-EphA4 S-20, 1:1000; catalog \# sc-921, Santa Cruz Biotechnology; RRID:AB_2099367) as loading controls. Specie-specific horseradish peroxidase-conjugated secondary antibodies (GE Healthcare or Jackson ImmunoResearch) and a chemiluminiscent substrate (Millipore) were used to visualize the bands in a membrane image analysis system (Bio-Rad).

Stripe assay. MGE explants were cultured on stripes of $50 \mathrm{ng} / \mu \mathrm{l}$ of total recombinant protein prepared as previously described (Yamagishi et al., 2011). Samples were fixed with $4 \%$ sucrose $/ 4 \%$ PFA in PBS for $30 \mathrm{~min}$ at room temperature and washed and incubated with rabbit anti$\beta$ III-tubulin antibody (1:500; catalog \#ab18207, Abcam; RRID:AB_ 444319) after permeabilization in $0.2 \%$ Triton X-100/PBS. Alexa-488 donkey anti-rabbit antibody (Jackson ImmunoResearch) was used to visualize the $\beta$ III-tubulin signal. The percentage of $\beta$ III-tubulin + pixels over red stripes was quantified using ImageJ (automatic stripe assay analysis by Daniel del Toro), and calculated as repulsion index (applying the formula $y=-0,02 x+1$, which represents a value of 1 with $0 \%$ of green pixels over red stripes and a value of 0 with 50\%) and represented as fold change in relation to $\mathrm{Fc} / \mathrm{Fc}$ control stripes (relative repulsion index).

Experimental design and statistical analyses. Data were assessed for normality by the Kolmogorov-Smirnov test or the Shapiro-Wilk test and for homoscedasticity by Levene's test. The two-tailed unpaired Student's $t$ test (Excel), one-way ANOVA [F(degrees of freedom between, degrees of freedom within)] with Tukey's corrected multiple comparison tests, or two-way ANOVA ( $p$ genotype and $p$ interaction) with Sidak's corrected multiple comparison tests (GraphPad Prism 7) were used to determine statistical differences between the means of two or more independent groups of animals, respectively. The number of independent values $(n)$ in each experiment was as follows: 12 controls versus 6 mutants (see Fig. $2 C$; three sections/animal), 6 controls versus 6 mutants (see Figs. 2D,E,H,I, $3 D-F, 7 C, D$; three sections/animal), 13 controls versus $4-5$ mutants (see Fig. $3 H$; three sections/animal), 3 controls versus 3 mutants (see Fig. 4B-F; 2-10 sections/animal), 4 controls versus 4 mutants (see Fig. $5 F-H$ ); three sections/animal), 6 controls versus 3 mutants (see Fig. $6 D$; 9 measures/explant, 1-6 explants/animal), and 38-45 explants/condition of 4-5 independent experiments (see Fig. $6 K, O$ ). The $p$ value in each experiment is indicated, and significance was considered when ${ }^{*} p<$ $0.05,{ }^{* *} p<0.01$, or ${ }^{* *} p<0.001$. Error bars were calculated using SEM.

\section{Results}

\section{FLRT2 and FLRT3 are expressed in the developing cortex}

FLRT2 and FLRT3 gene expression was studied by in situ hybridization in the murine telencephalon at E13.5 and E15.5, when extensive tangential migration of interneurons occurs. Positive expression included pallial and subpallial (P/SP) regions. FLRT2 was detected in the lateral ganglionic emminence (LGE), striatum, and CP of the developing cortex (Fig. 1 $A, B$ ). Instead, FLRT3 was detected in the VZ/SVZ region at the boundary between the LGE and MGE at E13.5 (Fig. 1C), globus pallidus (GP) and in upper parts of the CP and marginal zone (MZ) and in the IZ of the developing cortex at E15.5 (Fig. 1D). By using specific antibodies against the extracellular domain (ECD) of FLRT2 or FLRT3 on E14.5 brain sections, we confirmed the expression pattern observed by in situ hybridization (Fig. 1E, $H, K-N)$. However, FLRT2 immunofluorescence revealed a significant and graded expression of the protein (from apical low to basal high) within the IZ, which is probably recognizing FLRT2 in corticofugal/corticothalamic axons and/or the FLRT2 $^{\mathrm{ECD}}$ released from adjacent neurons in the CP (Fig. $1 F$, asterisk; Fig. 1O, summary; Yamagishi et al., 2011). On the other hand, FLRT3 immunofluorescence detected the expression of the protein in the IZ and in the upper part of the CP and the MZ, which displays a lateral (high) to dorsomedial (low) gradient along the cortical tangential axis (Fig. 1H,I). We costained these sections with $\mathrm{CB}$, a marker that labels a substantial fraction of cortical interneurons, specifically at these stages, $40 \%$ of cortical GABAergic EGFP+ neurons from $R 26 R$ $\mathrm{EYFP}^{\mathrm{lx} /+} ; \mathrm{Dl} \times 5 / 6-\mathrm{Cre}^{+}$brains were $\mathrm{CB}+$ and $94.8 \%$ of the $\mathrm{CB}+$ neurons coexpressed EGFP+ (Extended Data Fig. 2-1; Anderson et al., 1997). These FLRT/CB costainings revealed that the SP and the IZ/SVZ streams of cortical migrating interneurons 

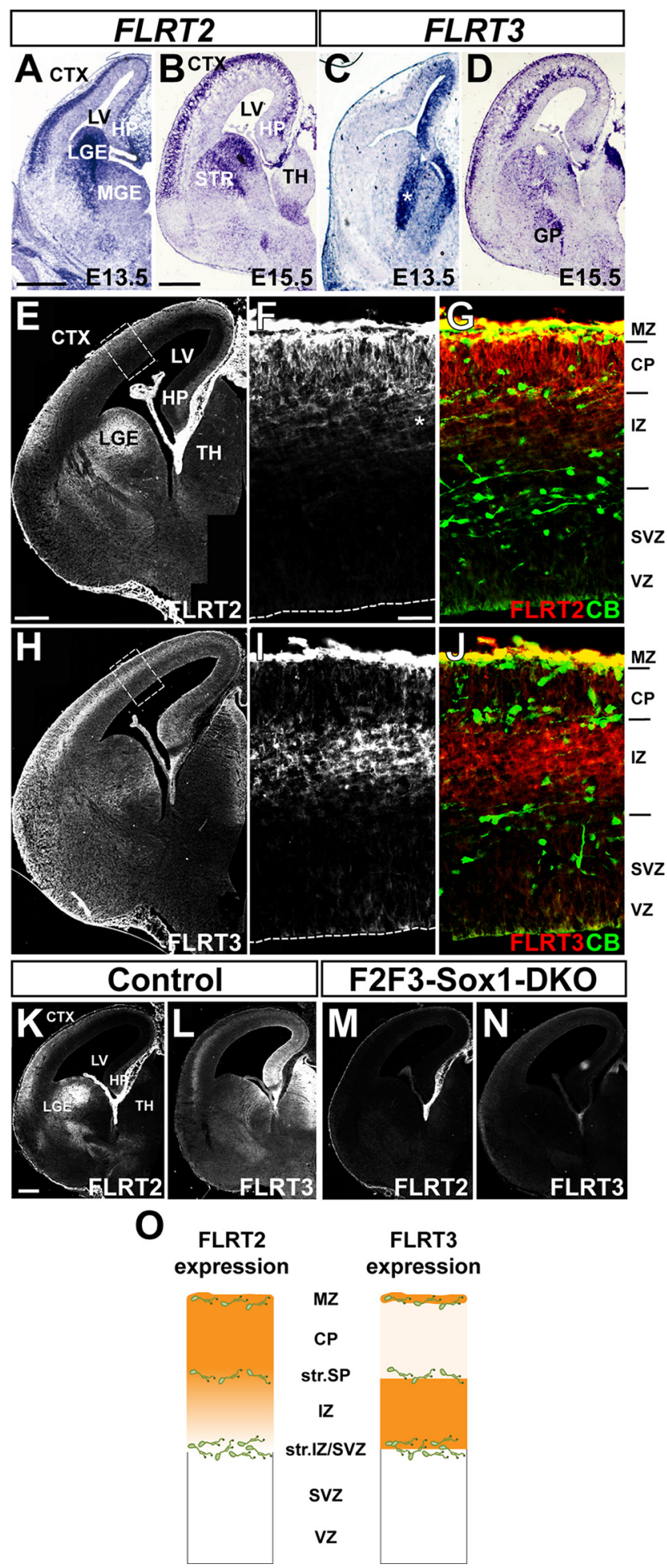

Figure 1. Expression pattern of FLRT2 and FLRT3 in the developing cortex of mouse embryos. $\boldsymbol{A}-\boldsymbol{D}$, In situ hybridization for FLRT2 $(\boldsymbol{A}, \boldsymbol{B})$ or FLRT3 $(\boldsymbol{C}, \boldsymbol{D})$ on coronal sections of embryonic mouse brain at E13.5 $(\boldsymbol{A}, \boldsymbol{C})$ and E15.5 $(\boldsymbol{B}, \boldsymbol{D})$. Only one hemisphere is shown. Asterisk in $\boldsymbol{C}$ indicates the boundary between LGE and MGE. $\boldsymbol{E}-\boldsymbol{J}$, Single immunofluorescence with specific antibodies against the ECD of FLRT2 $(\boldsymbol{E}, \boldsymbol{F})$ or FLRT3 $(\boldsymbol{H}, \boldsymbol{I})$ or in combination with $(B(\boldsymbol{G}, \boldsymbol{J})$, on coronal sections of embryonic mouse brain at E14.5. $(\boldsymbol{F}, \boldsymbol{G}, \mathbf{I}, \mathbf{J})$. High-power images of the cortex region indicated in $\boldsymbol{E}(\boldsymbol{F}, \boldsymbol{G})$ and $\boldsymbol{H}(\boldsymbol{I}, \boldsymbol{J})$ with a dashed rectangle. Asterisk in $\boldsymbol{F}$ highlights the expression of FLRT2 in the IZ. The boundaries between different zones are indicated on the left $(\boldsymbol{G}, \boldsymbol{J}) . \boldsymbol{K}-\boldsymbol{N}$, Immunofluorescence against FLRT2 $(\boldsymbol{K}, \boldsymbol{M})$ or FLRT3 $(\boldsymbol{L}, \boldsymbol{M})$ on coronal sections of embryonic brains (E14.5) from controls $(\boldsymbol{K}, \boldsymbol{L})$ and Sox1Cre conditional FLRT2;FLRT3 double knock-out embryos (F2F3-Sox1-DKO; $M, M$ ), demonstrating the specificity of the antibodies and the efficiency of the Sox1-Cre line in recombining avoided the regions of higher FLRT expression, in particular the IZ, where both proteins FLRT2 and FLRT3 colocalized (Fig. 1G, $J, O)$. These observations prompted us to investigate whether FLRT proteins could be involved in maintaining the integrity of the cortical migratory streams of interneurons.

\section{FLRT2;FLRT3 double knock-out embryos show abnormal distribution of cortical interneurons}

To assess the contribution of FLRTs in the regulation of interneuron migration within the developing cortex in vivo, we used knock-out alleles (FLRT2 $2^{\text {null }}$ and FLRT3 $\left.3^{\text {null }}\right)$ in combination with conditional alleles (FLRT2 $2^{l x}$ and FLRT3 $3^{l x}$ ) that were crossed with the whole nervous-system-specific Cre lines Sox1-Cre or NestinCre (Extended Data Fig. 2-2). From these crosses we obtained several single mutant embryos, including FLRT2 ${ }^{\text {null/lix }}$;Sox1-Cre+ (hereafter, F2-Sox1-KO), FLRT3 ${ }^{\text {null/lx }}$;Sox1-Cre+ (hereafter, F3Sox1-KO), and FLRT3 ${ }^{\text {null/lix }}$;Nestin-Cre+ (hereafter, F3-Nestin-KO), as well as double knock-out embryos FLRT2 ${ }^{\text {null/x }} ; F L R T 3^{\text {null/lx }}$;Sox1Cre+ (hereafter, F2F3-Sox1-DKO). All mutant embryos were obtained at expected Mendelian ratios, and littermates with other genotype combinations were pooled as controls because we did not observe significant differences in cortical interneuron distribution among these genotypes (data not shown). We first analyzed E14.5 mutant embryos by $\mathrm{CB}$ staining to quantify the total numbers of interneurons reaching the cortex as well as their relative distribution along the cortical wall. For the latter, a 10-bin grid was placed equidistant from the pallial-subpallial boundary and the dorsal part of the cortex on intermediate coronal sections (at the thalamic level), covering $\sim 30 \%$ of the cortical surface between these two landmarks (Fig. $2 A, B$ ). Then, the percentage of $\mathrm{CB}+$ neurons in each bin was calculated, from the upper $\mathrm{CP}$ to the $\mathrm{VZ}$ (Fig. $2 A, B$ ). This analysis revealed that in the F2-Sox1-KO, F3-Sox1-KO, or F3-Nestin-KO single knock-out embryos, the total abundance of $\mathrm{CB}+$ cortical interneurons, as well as their apical-to-basal distributions, were similar to that of controls, indicating that neither FLRT2 nor FLRT3 is necessary for interneurons to reach the developing cortex nor for the maintenance of the three migratory streams (Fig. 2C-E; Extended Data Fig. 2-3).

FLRT proteins exhibit a high degree of homology both in sequence and structure and display certain degrees of functional redundancy during the formation of different tissues, including the brain and heart (Lacy et al., 1999; Müller et al., 2011; Seiradake et al., 2014; del Toro et al., 2017) (see below, Discussion). Considering this evidence and the observation that FLRT2 and FLRT3 were coexpressed in the cortex, in particular in the IZ (Fig. $1 F, I$ ), we addressed the possibility that both proteins could have a redundant role in controlling intracortical distribution of interneurons. For this purpose, we performed the same $\mathrm{CB}$ staining-based quantification as above in the F2F3Sox1-DKOs at E14.5. Interestingly, the distribution of $\mathrm{CB}+$ cortical interneurons in the F2F3-Sox1-DKO embryos was significantly different from that of controls (Fig. $2 F-H$ ), whereas the total number of interneurons was unaffected (relative total cortical CB + cells \pm SEM: $1 \pm 0.077$ and $0.805 \pm 0.096$ in controls and mutants, respectively; $n=6$, three sections/animal, Student's $t$ test, $p=0.108)$. Quantification of the relative

both alleles in the same animal. $\mathbf{0}$, Summary of the protein distribution and levels (in orange) for FLRT2 and FLRT3 in the developing cortex of the mouse embryo at E14.5. Interneurons in the streams are drawn as green cells. Scale bar, $\boldsymbol{A}, \boldsymbol{B}, \boldsymbol{E}, 200 \mu \mathrm{m} ; \boldsymbol{F}, 20 \mu \mathrm{m} ; \boldsymbol{K}-\boldsymbol{N}, 250 \mu \mathrm{m}$. CTX, Cortex; STR, striatum; TH, thalamus; LV, lateral ventricle; HP, hippocampus. 

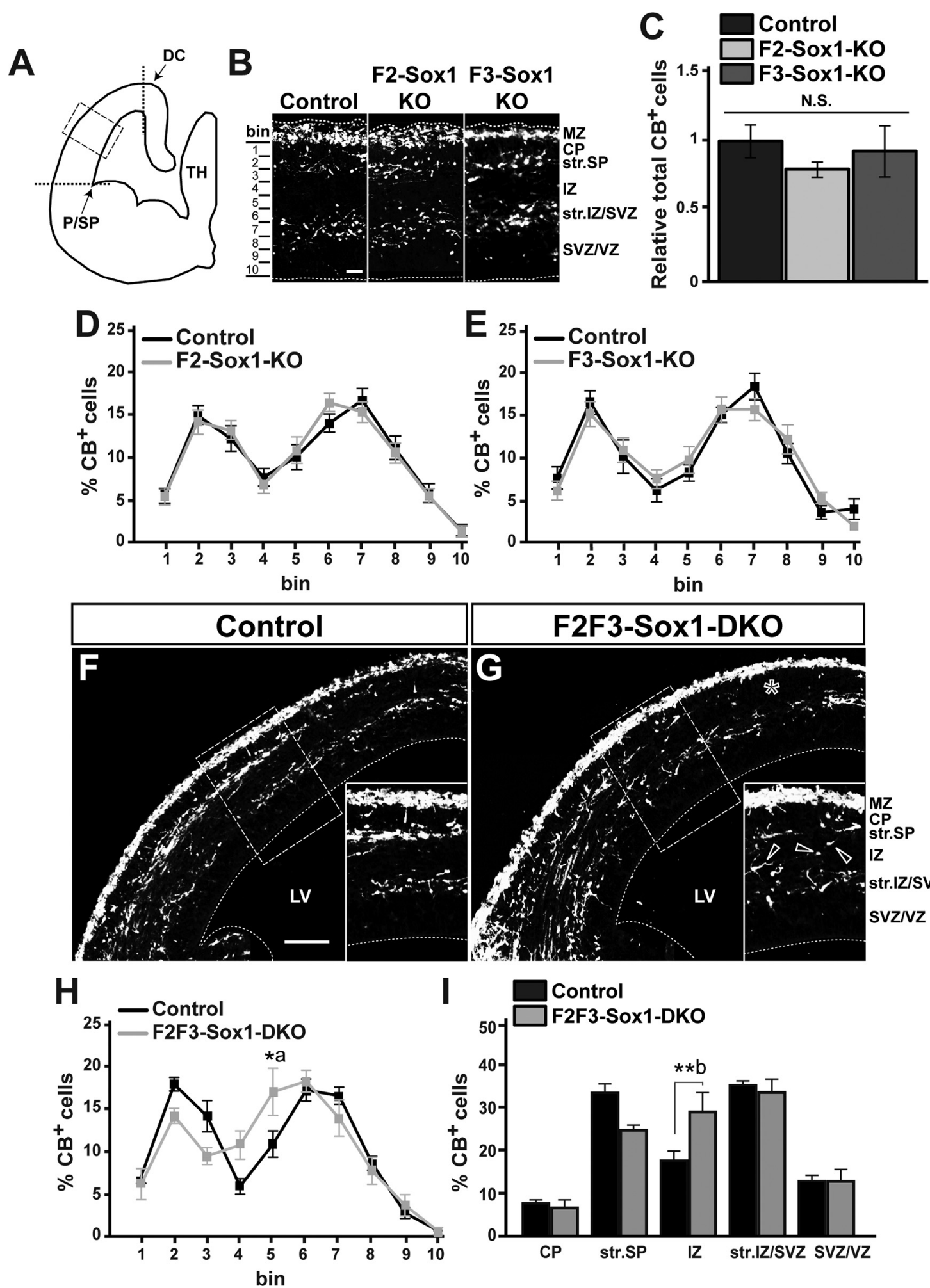

Figure 2. Disorganized tangential migratory streams of interneurons in the developing cortex of the double FLRT2; FLRT3 knock-out brains. $\boldsymbol{A}$, Diagram of a coronal section at intermediate regions in the rostro-caudal axis of an embryonic mouse brain at E14.5 (one hemisphere is shown). The landmarks DC (dorsal cortex) and P/SP and the region equidistant from DC and P/SP (dashed rectangle) that was considered for interneuron quantification are indicated. $\boldsymbol{B}$, Representative (B staining showing the distribution of cortical interneurons in either control, Sox1-Cre conditional FLRT2 or FLRT3 single knock-out embryos (F2-Sox1-K0 and F3-S0x1-K0, respectively). Includes the arbitrary subdivision of the cortical region depicted in $\boldsymbol{A}$ (dashed rectangle) in 10 equal bins (excluding the marginal zone) to assess $\mathrm{CB}+$ interneuron distribution along the cortical width. C, Quantification of the relative amount of CB+ interneurons in Sox1-Cre conditional FLRT2 or FLRT3 single knock-out embryos (F2-Sox1-KO and F3-Sox1-KO, respectively; gray bars) between the DC and the P/SP $A$, Values are expressed as the mean fold change to control (black bar) $\pm \operatorname{SEM}\left(n=6-12\right.$ animals per genotype, 3 sections/animal). N.S., nonstatistical differences (1-way ANOVA; $\left.F_{(2,21)}=2.411, p=0.1141\right)$. D, E, Quantification of the percentage of CB + cortical interneurons in each bin $(\boldsymbol{B})$ in controls (black lanes) and in Sox1-Cre conditional FLRT2 (D) or FLRT3 (E) knock-out embryos (F2-Sox1-K0 and F3-Sox1-KO, respectively; gray lanes). Values are expressed as the mean \pm SEM ( $n=6$ animals per genotype, 3 sections/animal). No statistical differences for each bin (2-way ANOVA; $\boldsymbol{D}, p$ genotype $>0.9999, p$ interaction $=0.9324 ; \boldsymbol{E}, p$ genotype $>0.9999, p$ interaction $=0.6014)$ were found. $\boldsymbol{F}, \boldsymbol{G}$, Representative $(B$ staining showing the distribution of cortical interneurons in control $(\boldsymbol{F})$ and in the Sox1-Cre conditional FLRT2/FLRT3 double knock-out embryos (F2F3-Sox1-DKO, $\mathbf{G}$ ) at E14.5. Insets, High-power images of the corresponding regions depicted with a dashed rectangle. Asterisk in $\mathbf{G}$ indicates the reduction of CB+ 
distribution of $\mathrm{CB}+$ interneurons along the cortical wall showed a reduction of interneurons in upper bins concomitant with a significant increase in middle bins in the F2F3-Sox1-DKOs (six controls vs six mutants, three sections/animal; two-way ANOVA, $p$ genotype $>0.9999, p$ interaction $=0.0022$; bin 5 : control mean \pm SEM: $10.67 \% \pm 1.67$, DKO mean \pm SEM: $16.66 \% \pm 2.96$; Sidak's-corrected multiple comparison test, $p=0.0212$ ), whereas the deeper bins were not affected (Fig. $2 H$ ). By grouping the bins in morphologic cortical layers, we realized that interneurons redistributed from the SP stream (upper bins) toward the IZ region underneath (two-way ANOVA, $p$ genotype $>0.9999, p$ interaction $=0.0032$; IZ control mean \pm SEM: $16.49 \% \pm 2.33 ; \mathrm{DKO}$ mean \pm SEM: $27.21 \% \pm 4.73$; Sidak's corrected multiple comparison test, $p=0.0073$ ), whereas the relative numbers of $\mathrm{CB}+$ interneurons in the IZ/SVZ stream were normal (Fig. 2I). In addition, quantification of the $\mathrm{CB}+$ signal specifically at the $\mathrm{MZ}$ did not reveal significant differences among controls and the single $\mathrm{KOs}$ (data not shown) or the F2F3-Sox1-DKOs, both in the number of $\mathrm{CB}+$ cells in the MZ (total CB + cells \pm SEM in the MZ: 122,33 \pm 11.91 and $137.83 \pm 10.38$ in controls and mutants, respectively; $n=6$, three sections/animal, Student's $t$ test, $p=0.308$ ) as well as their distribution along the cortex (Fig. $3 A-D$ ). Importantly, these defects in interneuron distribution were not related to a general defect in the cortical cyto architecture as the size of the cortex as well as the layering pattern, analyzed with several specific markers such as Tbr1, Ctip2, or Satb2, were normal in the F2F3-Sox1DKOs (Extended Data Fig. 2-4).

Next, we reasoned that if $\mathrm{CB}+$ interneurons in the SP stream were abnormally located within the IZ in the double mutants, then the number of $\mathrm{CBs}+$ migrating toward more dorsal cortical regions should be reduced and the length of the SP stream shortened. To test this possibility, we first quantified the number of $\mathrm{CB}+$ interneurons along the tangential axis of the cortex at three different levels, proximal, medial, and distal from the pallium/ subpallium boundary (regions I, II, and III, respectively; Fig. $3 A$ ). Indeed, we noticed that the F2F3-Sox1-DKOs displayed significantly fewer $\mathrm{CB}+$ interneurons at the most distal level compared with controls (Fig. 3E; six controls vs six mutants, three sections/animal; two-way ANOVA, $p$ genotype $>0.9999$, $p$ interaction $=0.0133$; region III: control mean \pm SEM: $33.59 \% \pm$ 2.04, DKO mean \pm SEM: $22.42 \% \pm 5.85$; Sidak's corrected multiple comparison test, $p=0.0464$; compare Fig. $2 F, G$, asterisk region). Interestingly, an overall significant reduction of $\mathrm{CB}+$ interneurons was found along the width of the cortex in region III, including upper layers, the ones mainly contributed by the SP stream (Fig. 3F; six controls vs six mutants, three sections/animal; two-way ANOVA, $p$ genotype $=0.0084, p$ interaction $=$

\footnotetext{
interneurons in the SP stream of the mutants at more dorsal cortical regions. Inset, Open arrowheads $(\boldsymbol{G})$ point to $C B+$ interneurons within the $\mathrm{IZ}$ in the mutants. $\boldsymbol{H}, \boldsymbol{I}$, Quantification of the percentage of $\mathrm{CB}+$ cortical interneurons in each bin $(\boldsymbol{H})$ and in each morphologic layer $(\boldsymbol{I})$ in control (black lane/bars) and in Sox1-Cre conditional FLRT2;FLRT3 double knock-out embryos (F2F3-Sox1-DK0, gray lane/bars). Values are expressed as the mean \pm SEM ( $n=6$ animals per genotype, 3 sections/animal). Statistical differences (2-way ANOVA; $\boldsymbol{H}, \boldsymbol{p}$ genotype $>$ $0.9999, p$ interaction $=0.0022 ; \boldsymbol{I}, p$ genotype $>0.9999, p$ interaction $=0.0032$ ) are indicated, after Sidak's corrected multiple comparison: ${ }^{* a} p=0.0212,{ }^{*}{ }^{* b} p=0.0073$. An assessment of the specificity of $C B$ as an interneuron marker during cortex development and of the recombination efficiency of Nestin-Cre and Sox1-Cre lines are shown in Extended Data Figures 2-1 and $2-2$, respectively. Analysis of the effects of single FLRT3 deletion on cortical interneuron distribution during development and of the morphology and layering of the embryonic cortex in the F2F3-Sox1-DK0s are shown in Extended Data Figures 2-3 and 2-4, respectively. Scale bar, $\boldsymbol{B}$, $50 \mu \mathrm{m} ; \boldsymbol{F}, 100 \mu \mathrm{m}$. TH, thalamus; DC, dorsal cortex; P/SP, pallium/subpallium boundary.
}

$\leftarrow$
0.1904). Second, we measured the length of the SP stream as a percentage of the total length of the cortex from lateral to dorsal as described in Figure $3 G$. As we expected, the length of the SP stream was significantly reduced in the F2F3-Sox1-DKOs but not in the single mutants [Fig. $3 H$; 13 controls vs $4-5$ mutants, three sections/animal; one-way ANOVA subplate stream (str. SP), $F_{(3,14)}=4.212, p=0.0256$; str.SP control, mean \pm SEM: $69.09 \% \pm 2.509 ; \mathrm{F} 2-\mathrm{S}$ ox1-KO mean \pm SEM: $58.18 \% \pm 1.892$; F3-Sox1-KO mean \pm SEM: $60.28 \pm 1.352$; F2F3-Sox1-DKO mean \pm SEM: $45.67 \pm 7.280$; Tukey's corrected multiple comparison test, $p=0.0154$ for control vs F2F3-Sox1-DKO; compare Fig. $2 F, G$, asterisk region]. In contrast, the length of the IZ/SVZ stream in the double mutants was similar to controls, suggesting that the effects of the double FLRT2;FLRT3 deletion were specific for the $\mathrm{CB}+$ interneurons of the SP stream (Fig. $3 H$ ). In summary, our analysis shows that FLRT2 and FLRT3 have functionally redundant roles in mediating the proper distribution of interneurons during cortical development, in particular for those located in the SP stream.

\section{Postnatal FLRT2;FLRT3 double knock-out animals display abnormal distribution of SST + interneurons}

To gain insight into the possible functional relevance of our observations during development for the mature brain, we analyzed the distribution of interneurons in postnatal F2F3-Sox1DKO brains when the formation of the cortical structure and neuron connectivity is already accomplished. The diversity of adult GABAergic interneurons can be reduced to three main groups that account for the vast majority of all inhibitory neurons, according to the expression of the calcium-binding protein $\mathrm{PV}$, the neuropeptide SST, and the inotropic serotonin receptor 3A (Rudy et al., 2011). Within these groups, CR and VIP delineate specific subtypes (Gonchar and Burkhalter, 1997; Lodato et al., 2011; Rudy et al., 2011; Pfeffer et al., 2013). Using specific antibodies (CR, PV, and SST) or RNA probes (VIP) against each of these markers, we performed immunofluorescence or in situ hybridization studies on fixed brains at P21 of F2F3-Sox1-DKOs and control littermates and quantified the distribution of the different types of interneurons in the cortex in an arbitrary 10-bin grid (Fig. 4). Then, the percentage of positive neurons in each bin was calculated for each animal. The results showed that the relative layer distribution of SST + interneurons was abnormal in the F2F3-Sox1-DKOs, displaying a subtle but significant shift in deeper layers (Fig. 4A-C; three controls vs three mutants, 210 sections/animal; $B$, two-way ANOVA, $p$ genotype $>0.9999$, $p$ interaction $<0.0001$; bin 6: control mean \pm SEM: $13.33 \% \pm$ 1.14, DKO mean \pm SEM: $6.33 \% \pm 1.66$; bin 7: control mean \pm SEM: $14.90 \% \pm 1.38$, DKO mean \pm SEM: $8.54 \% \pm 0.18$; bin 9 : control mean \pm SEM: $12.92 \% \pm 0.39$, DKO mean \pm SEM: $19.85 \% \pm 2.4$; Sidak's corrected multiple comparison test for bin 6 , bin 7, and bin 9, $p=0.0064, p=0.0171$, and $p=0.0071$, respectively; $C$, two-way ANOVA, $p$ genotype $=0.3589, p$ interaction $=$ 0.0005; L.5 control mean \pm SEM: $28.23 \% \pm 2.52$; DKO mean \pm SEM: $14.88 \% \pm 1.82$; L. 6 control mean \pm SEM: $37.45 \% \pm 1.46$; DKO mean \pm SEM: $50.68 \% \pm 3.9$; Sidak's corrected multiple comparison test for L.5 and L.6, $p=0.001, p=0.028$, respectively), whereas the distribution of $\mathrm{PV}+, \mathrm{CR}+$, and $\mathrm{VIP}+$ interneurons was not altered (Fig. $4 D-F$ ). Therefore, these observations suggest that FLRT2 and FLRT3 were required for the proper final positioning of a particular subset of SST+ interneurons. Most probably these are non-Martinotti cells as Martinotti SST + interneurons migrate preferentially through the $\mathrm{MZ}$, and no defects in interneuron distribution in this 

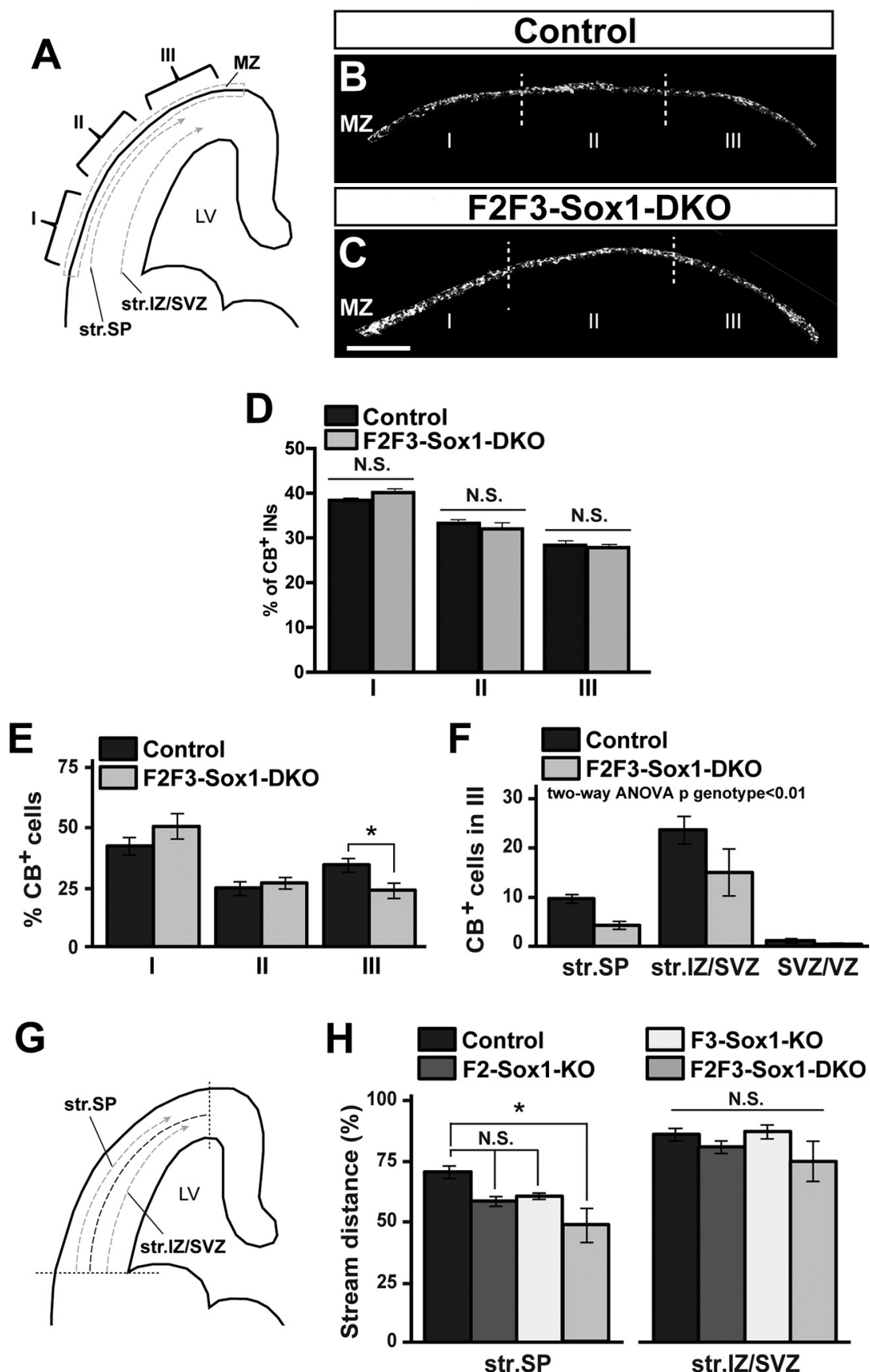

Figure 3. Interneuron distribution defects in the FLRT2 and FLRT3 double knockouts mainly affect the subplate stream. $\boldsymbol{A}$, Diagram of the developing cortex of a E14.5 mouse embryo showing the three regions (I, II, III) where CB+ interneurons were quantified along the tangential axis. $\boldsymbol{B}, \boldsymbol{C}$, ImageJ cropped representative images of the MZ stained with CB from control (top) and from Sox1-Cre conditional FLRT2; FLRT3 double knock-out embryos (F2F3-Sox1-DK0, bottom). The regions I, II, and III in $\boldsymbol{A}$ are indicated. $\boldsymbol{D}$, Quantification of the percentage of CB + cells in each region (I-III) of the MZ in control (black bar) and in Sox1-Cre conditional FLRT2;FLRT3 double knock-out embryos (F2F3-Sox1-DK0, gray bar). Values are expressed as the mean \pm SEM ( $n=6$ animals per genotype, 3 sections/animal). N.S., Nonstatistical differences (2-way ANOVA; $p$ genotype $>0.9999, p$ interaction $=0.0757$ ). $\boldsymbol{E}$, Quantification of the percentage of $C B+$ cortical interneurons in regions I, II, and III $(\boldsymbol{A})$ in controls (black bars) and in the Sox1-Cre conditional FLRT2; FLRT3 double knock-out embryos (F2F3-Sox1-DK0, gray bars). Values are expressed as the mean \pm SEM ( $n=6$ animals per genotype, 3 sections/animal). Statistical differences (2-way ANOVA; $p$ genotype $>0.9999, p$ interaction $=$ 0.0133 ) are indicated after Sidak's corrected multiple comparison: ${ }^{*} p=0.0464$. F, Quantification of the number of $(B+$ cortical interneurons in superficial (str.SP), middle (str.IZ/SVZ), and deeper (SVZ/NZ) parts along the cortical width in region III in control (black bars) and in Sox1-Cre conditional FLRT2;FLRT3 double knock-out embry0s (F2F3-Sox1-DKO, gray bars). Values are expressed as the mean \pm SEM ( $n=6$ animals per genotype, 3 section/animal). Statistical differences (2-way ANOVA; $p$ genotype $=0.0084, p$ interaction $=0.1904)$. $\mathbf{G}$, Diagram of the developing cortex of a E14.5 mouse embryo showing the quantification criteria to assess the distance of the SP and IZ/SVZ streams (str.SP and str.IZ/ SVZ, respectively; gray dashed arrows), relative to the total length of the cortex (dashed black line). $\boldsymbol{H}$, Relative stream region were observed in the F2F3-Sox1DKO embryos (Fig. 3A-D; Lim et al., 2018).

Noncell autonomous function of FLRTs during interneuron development

Previous reports have shown that FLRT functions involve cell-autonomous (as coreceptors) as well as noncell-autonomous (as ligands) mechanisms of action (Yamagishi et al., 2011; O’Sullivan et al., 2012; Leyva-Díaz et al., 2014; Seiradake et al., 2014). In this regard, the expression of FLRT2 and FLRT3 by migrating excitatory neurons in the cortex (Fig. 1) would suggest a regulation of interneuron distribution by a noncell-autonomous mechanism. Indeed, stimulation of MGE explant-derived interneurons in vitro with either FLRT2 or FLRT3 ECDs fused to Fc revealed a significant binding of these recombinant proteins to the cell surface of interneurons, arguing for the presence of membrane receptors able to bind FLRTs and eventually initiate a signaling cascade (Fig. 5A-C). However, we also detected significant expression of FLRT2 and FLRT3 in interneurons in vivo and in vitro that can account for some cell-autonomous functions of these proteins (data not shown). To sort out this question, we used the Emx1Cre line, whose expression in the cortex is restricted to cortical neurons excluding subpallial-derived interneurons (Chan et al., 2001; Gorski et al., 2002; Extended Data Fig. 5-1). Thus, we generated FLRT2 $2^{\text {null/lx}}$; FLRT3 ${ }^{\text {null/lx }}$;Emx1-Cre ${ }^{+}$embryos (hereafter, F2F3-Emx1-DKO) and analyzed the distribution of cortical interneurons by $\mathrm{CB}$ staining as described above. We noticed a different distribution of $\mathrm{CB}+$ interneurons within the cortex of these mutants compared with controls (Fig. 5D-F; F, four controls vs four mutants, three sections/animal; two-way ANOVA, $p$ genotype $>0.9999, p$ interaction $<0.005$; bin 3: control mean \pm SEM: $15.30 \% \pm 2.10$; DKO mean \pm SEM: $8.02 \% \pm 1.78$; Sidak's corrected multiple comparison test, $p=0.0073$ ), whereas the total number of $\mathrm{CB}+$ cortical interneurons was unaltered (relative total cortical $\mathrm{CB}+$

length (str.SP, left columns; str.IZ/SVZ, right columns) to the total length of the cortex in controls (black bars), in Sox1-Cre conditional single FLRT2 (F2-Sox1-KO) or FLRT3 (F3-Sox1-K0) and FLRT2;FLRT3 double knock-out embryos (F2F3-Sox1-DK0; gray bars), as indicated. Values are expressed as the mean \pm SEM ( $n=4-13$ animals per genotype, 3 sections/animal). Statistical differences (1-way ANOVA; str.SP $F_{(3,14)}=4.212, p=$ $0.0256)$ are indicated, after Tukey's corrected multiple comparison: ${ }^{*} p=0.0154$. N.S., Nonstatistical differences (1-way ANOVA; str.SP, after Tukey's corrected multiple comparison: $p=2423$; control vs F2-Sox1-KO, $p=0.3696$; control vs F3-Sox1-K0, $p=$ 0.9918; F2-Sox1-K0 vs F3-Sox1-K0; str.IZ/SVZ $F_{(3,14)}=0.997$, $p=0.423$ ). Scale bar, $200 \mu \mathrm{m}$. LV, lateral ventricle. 

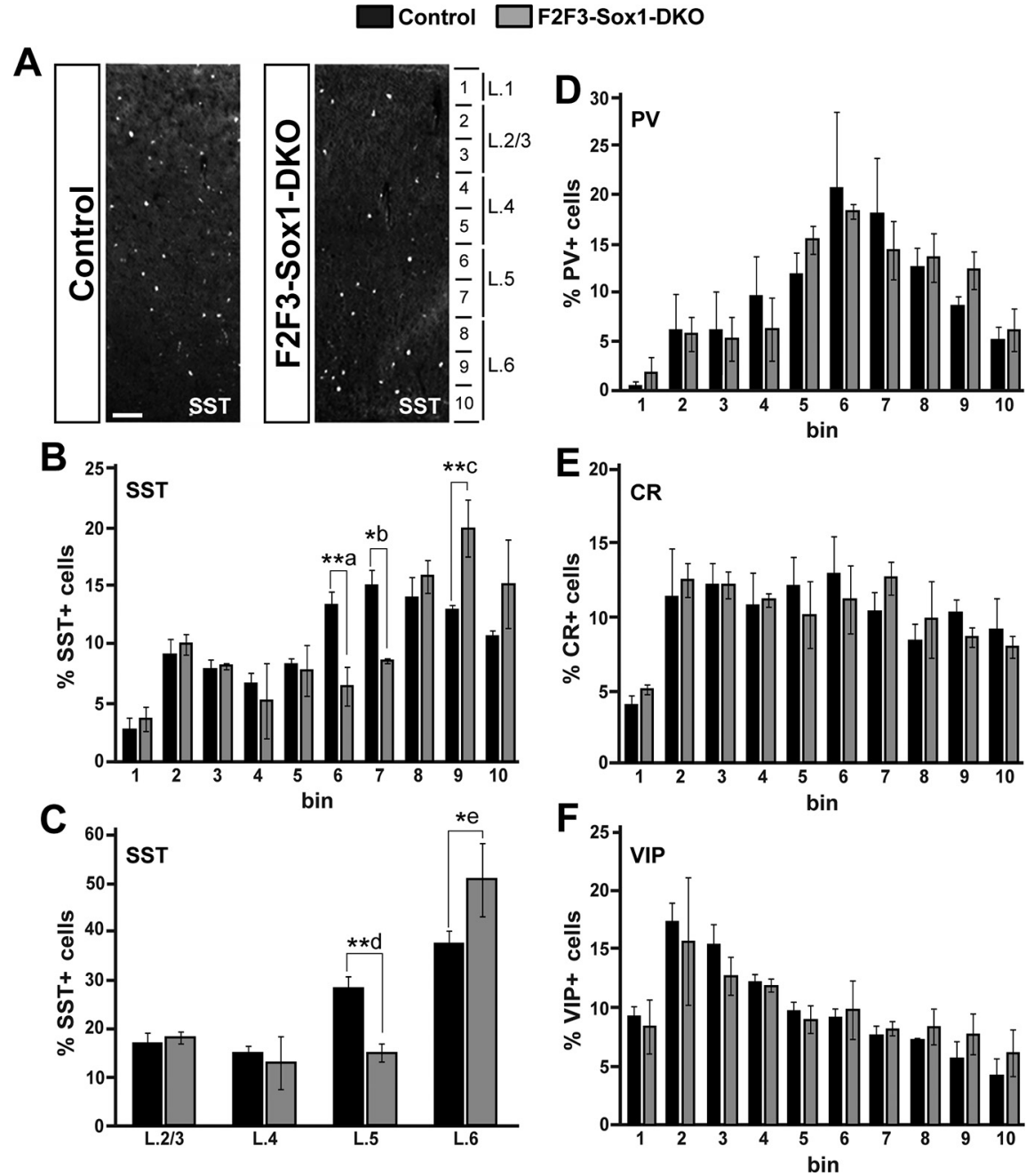

Figure 4. Abnormal layer distribution of SST+ interneurons in postnatal FLRT2;FLRT3 double knock-out brains. $\boldsymbol{A}-\boldsymbol{F}$, Distribution of SST $+(\boldsymbol{A}-\boldsymbol{C}), \mathrm{PV}+(\boldsymbol{D}), \mathrm{CR}+(\boldsymbol{E})$, and VIP+ $(\boldsymbol{F})$ interneurons through the cortical wall of the dorsal cortex in coronal sections (between positions 1 and -2.5 around bregma) of brains obtained from P21 F2F3-Sox1-DK0 (gray bars) and control animals (black bars). Quantification was performed within 10 arbitrary bins covering the dorsal part of the cortex including the cingulate/RSD (retrospenial dysgranular) cortex, primary and secondary motor cortex, primary somatosensory cortex, and primary and secondary visual cortex (Franklin and Paxinos, 2008). The data are represented as the percentage of each subtype of interneuron in each bin $(\boldsymbol{B}, \boldsymbol{D}-\boldsymbol{F} ; \boldsymbol{A})$. The percentage of SST+ interneurons in each anatomic layer in the $\mathrm{CP}$ (depicted in $\boldsymbol{A})$ is illustrated in $C$. Values are expressed as the mean \pm SEM ( $n=3$ animals per genotype, $2-10$ sections/animal). Statistical differences (2-way ANOVA; $\boldsymbol{B}, p$ genotype $>0.9999, p$ interaction $<0.0001 ; \boldsymbol{C}, p$ genotype $=0.3589, p$ interaction $=0.0005$ ) are indicated, after Sidak's corrected multiple comparison: ${ }^{* * a} p=0.0064,{ }^{* b} p=0.0171,{ }^{* * c} p=0.0071,{ }^{* * * \mathrm{~d}} p=0.0010,{ }^{* \mathrm{e}} p=0.028$. Nonstatistical differences were found in $\boldsymbol{D}-\boldsymbol{F}$ (2-way ANOVA; $\boldsymbol{D}, p$ genotype $>0.9999, p$ interaction $=0.8555 ; \boldsymbol{E}, p$ genotype $>$ $0.9999, p$ interaction $=0.8102 ; \boldsymbol{F}, p$ genotype $>0.9999, p$ interaction $=0.8630$ ). Scale bar, $\boldsymbol{A}, 100 \mu \mathrm{m}$.

cells \pm SEM: $1 \pm 0.023$ and $1.04 \pm 0.049$ in controls and mutants, respectively; $n=4$, three sections/animal; Student's $t$ test $p=$ 0.468). Indeed, the abnormal distribution of interneurons in these mutants shared the main features observed in the F2F3-Sox1DKOs: (1) significantly, fewer interneurons in the SP stream associated with an increase of interneurons within the IZ (Fig. $5 F, G ; G$, four controls vs four mutants, three sections/animal; two-way ANOVA, $p$ genotype $=0.6556, p$ interaction $=0.002$; str.SP control mean \pm SEM: $33.44 \% \pm$ 2.59; DKO mean \pm SEM: $24.72 \% \pm$ 1.35; Sidak's corrected multiple comparison test, $p=0.0071)$; (2) a shorter SP stream (Fig. $5 H$; four controls vs four mutants, three sections/animal; control mean \pm SEM: $69.43 \% \pm 1.67$; DKO mean \pm SEM: $55.26 \% \pm 4.67$; Student's $t$ test $p=0.0164)$, and (3) no effect on the IZ/SVZ stream (Fig. 5G,H). Together, these results strongly indicate that cortical interneuron distribution is regulated by FLRT2 and FLRT3 in a noncell-autonomous manner.
FLRT2 and FLRT3 display repulsive effects on migrating interneurons Interneurons derived from MGE explant cultures display an amazing intrinsic capacity to migrate, so they can be used to address several questions about migration mechanisms in vitro (Wichterle et al., 1999; Nery et al., 2002). Using these cultures, we first compared the migration distance of interneurons from MGEs isolated from F2F3-Sox1-DKOs and controls in Matrigel and observed no differences, suggesting that the absence of FLRT2 and FLRT3 does not impair the ability of interneurons to migrate out of the explant (Fig. 6A-D). Second, we addressed the functional effects of FLRT2 and FLRT3 on interneurons using a stripe assay where the cells migrating out of a MGE explant were given the choice to grow over an inert protein substrate $(\mathrm{Fc})$ or over a substrate containing FLRT ECDs fused to Fc (Fig. 6E,F). In control experiments using the same Fc protein for coating both stripes, interneurons did not show any growth preference and dispersed homogenously around the explant (Fig. $6 F, G$ ). In this case the percentage of anti- $\beta$ III-tubulin staining on red stripes was close to $50 \%$, and thus the normalized repulsive index equals 1 (Fig. $6 K$, dashed line). However, interneurons consistently avoided stripes that included recombinant FLRT $^{\mathrm{ECD}}$-Fc (Fig. $\left.6 F, H, I\right)$. In this case the relative repulsive index was $>1$, with statistical differences between FLRTs and Fc (Fig. $6 K$; 40-45 explants/condition of 4 independent experiments; one-way ANOVA $F_{(3,163)}=17.01, p<0.0001 ; \mathrm{Fc}$ mean \pm SEM: $1 \pm 0.112$; FLRT2 mean \pm SEM: $1.80 \pm 0.125$; FLRT3 mean \pm SEM: $2.18 \pm 0.213$; FLRT2 +3 mean \pm SEM: $2.60 \pm 0.190$; Tukey's corrected multiple comparison test, $p=0.003$ for $\mathrm{Fc} /$ FLRT2 ${ }^{\mathrm{ECD}}$-FC and $p<0.0001$ for $\mathrm{Fc} /$ $\mathrm{FLRT}^{\mathrm{ECD}}$-Fc). Interestingly, the combination of both FLRT2 ${ }^{\mathrm{ECD}}-\mathrm{FC}$ and $\mathrm{FLRT}^{\mathrm{ECD}}-\mathrm{FC}$ in the same red stripe induced a significant additive repulsive effect (Fig. 6J,K; one-way ANOVA $F_{(3,163)}=17.01, p<0.0001$; Tukey's corrected multiple comparison test $p<0.0001$ for Fc/FLRT2 $2^{\mathrm{ECD}}-\mathrm{Fc}+\mathrm{FLRT}{ }^{\mathrm{ECD}}-\mathrm{Fc}$ and $p=0.0033$ for FLRT2 ${ }^{\mathrm{ECD}}-\mathrm{Fc} / \mathrm{FLRT} 2^{\mathrm{ECD}}-\mathrm{Fc}+\mathrm{FLRT}^{\mathrm{ECD}}-\mathrm{Fc}$ ). These in vitro results demonstrated that FLRTs exert a repulsive effect on migrating interneurons.

\section{Unc5B/Ds are candidate FLRT receptors for interneuron repulsion}

Next, we addressed the question of what could be the receptors that bind FLRT2 and FLRT3 and mediate their repulsive effects on interneurons. Previous data lead us to focus on the Netrin receptors Unc5 (Unc5A-D), in particular Unc5B and Unc5D, as (1) Unc5 receptors mediate the repulsive effects of Netrin in different cell types, including neurons (Moore et al., 2007); (2) 


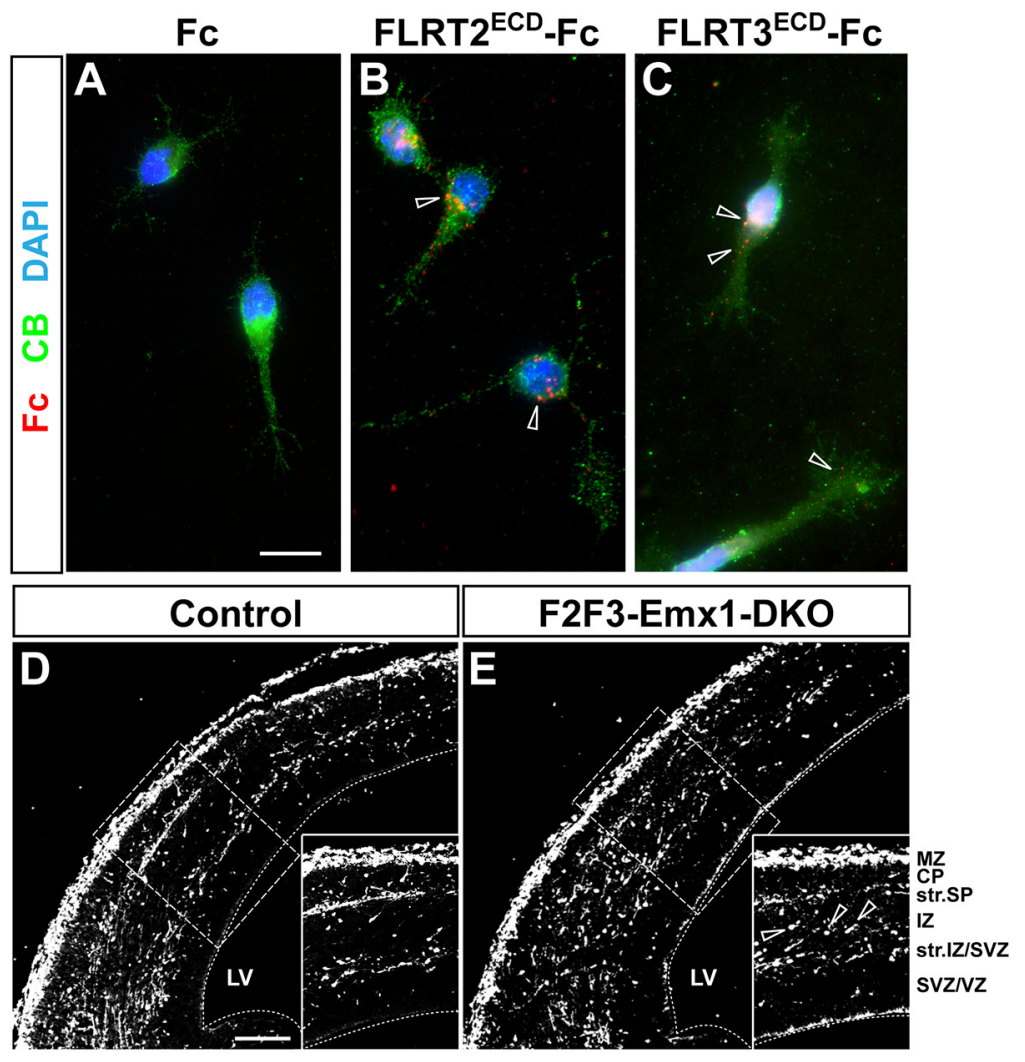

$\mathbf{F}$

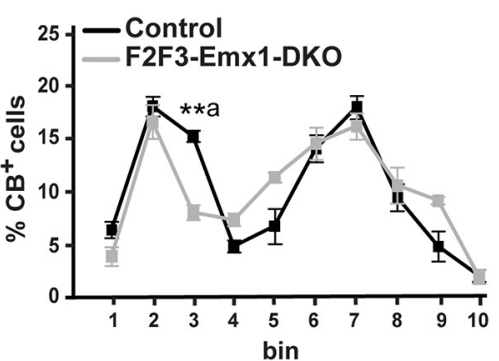

G

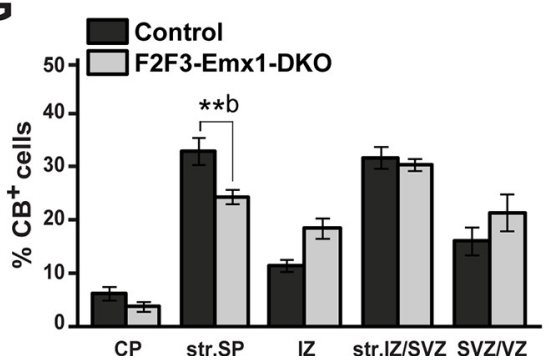

H

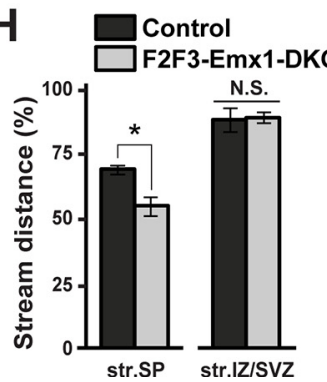

Figure 5. FLRT2 and FLRT3 exert a non cell-autonomous effect on the organization of the cortical interneuron streams during cortex development. $\boldsymbol{A}-\boldsymbol{C}$, MGE-derived interneurons were cultured in vitro for $48 \mathrm{~h}$ and then stimulated as indicated with recombinant $\mathrm{FC}(\boldsymbol{A})$, $\mathrm{FLRT}^{\mathrm{ECD}}-\mathrm{FC}(\boldsymbol{B})$ or $\mathrm{FLRT}^{\mathrm{ECD}}-\mathrm{FC}(\boldsymbol{C})$ proteins. After stimulation, cultures were fixed, processed for immunofluorescence against $F c$ in red (without permeabilization), $C B$ in green (after permeabilization), and counterstained with DAPI in blue. Open arrowheads point to clusters of FLRT2 (B) or FLRT3 (C) recombinant proteins on the surface of $C B+$ interneurons. $D, E$, Representative $C B$ staining showing the distribution of cortical interneurons in controls $(\boldsymbol{A})$ and in the Emx1-Cre conditional FLRT2; FLRT3 double knock-out embryos (F2F3-Emx1-DK0, $\boldsymbol{B}$ ) at E14.5. Insets, High-power images of the corresponding regions depicted with a dashed rectangle. Inset, Open arrowheads $(\boldsymbol{B})$ point to $C B+$ interneurons within the $I Z$ in the mutants. $\boldsymbol{F}, \boldsymbol{G}$, Quantification of the percentage of $C B+$ cortical interneurons in each bin $(\boldsymbol{D})$ and in each morphologic layer $(\boldsymbol{E})$ in controls (black lane/bars) and in Emx1-Cre conditional FLRT2;FLRT3 double knock-out embryos (F2F3-Emx1-DK0, gray lane/bars). Values are expressed as the mean \pm SEM ( $n=4$ animals per genotype, 3 sections/animal). Statistical differences (2-way ANOVA; $\boldsymbol{F}, p$ genotype $>0.9999, p$ interaction $=0.005 ; \boldsymbol{G}, p$ genotype $=0.6556, p$ interaction $=0.002$ ) are indicated, after Sidak's corrected multiple comparison: ${ }^{* a \mathrm{a}} p=0.0073,{ }^{* * \mathrm{~b}} p=0.0071$. $\boldsymbol{H}$, Percentage of stream distance (str.SP, left columns; str.IZ/SVZ, right columns) relative to the total length of the cortex (Figure 4D) in controls (black bars) and in Emx1-Cre conditional FLRT2;FLRT3 double knock-out embryos (F2F3-Emx1-DK0, gray bars).
FLRT2 ${ }^{\mathrm{ECD}}$ and FLRT3 ${ }^{\mathrm{ECD}}$ have been reported to bind Unc5B and Unc5D and trigger repulsion in neurons and in endothelial cells (Yamagishi et al., 2011; Seiradake et al., 2014); and (3) the Sip1 transcription factor, which is highly enriched in migrating cortical interneurons, is essential for the correct cortical interneuron guidance during development by fine-tuning the expression levels of Unc5B (van den Berghe et al., 2013). The part of the extracellular domain of FLRTs responsible for the interaction with Unc5 receptors has been recently mapped and mutated to generate specific Unc5 binding mutants (Seiradake et al., 2014). We used these mutant recombinant proteins to address the role of the FLRT-Unc5 interaction for the chemorepulsive effect of FLRTs on interneurons. Given the higher repulsive effect for FLRT3 (Fig. 6K), we used a FLRT3 mutant recombinant protein unable to bind Unc5 $\left(\mathrm{FLRT}_{3}{ }^{\mathrm{UF}}\right.$ ) in stripe assays (Seiradake et al., 2014). In these experiments a different batch of recombinant proteins was used, which triggered a stronger repulsive effect (compare Fig. 6K,O; Seiradake et al., 2014). As is shown in Figure $6 L-$ $O$, the impairment of Unc5 binding reduced significantly the repulsion triggered by FLRT3 on cultured interneurons, suggesting that FLRT3Unc5 interaction is involved in the repulsive response (Fig. 6O; 38-45 explants/condition of five independent experiments; one-way ANOVA $\left.F_{(2,121}\right)=47.17, p<0.0001 ;$ Fc mean \pm SEM: $1 \pm$ 0.134; FLRT3 mean \pm SEM: 3,64 \pm 0.248; FLRT3 $^{\text {UF }}$ mean \pm SEM: $2.93 \pm 0.202$; Tukey's corrected multiple comparison test, $p=0.0324$ for FLRT3-Fc/FLRT3 ${ }^{\mathrm{UF}}$-Fc).

To obtain genetic evidence for Unc5 receptors in interneuron guidance, we analyzed the cortex of E14.5 double Unc5B;Unc5D knockout embryos (Unc5D ${ }^{\text {null/null }} ;$ Unc5 $5 B^{\text {lx/null }}$;Nestin-Cre ${ }^{+}$; hereafter, Unc5B/D-DKO) with $\mathrm{CB}$ antibodies and analyzing interneuron distribution in these mutants, as above (Fig. 7A,B). The quantification of these experiments revealed that there were significantly fewer interneurons in the SP stream and more in the IZ of the Unc5B/D-DKOs, whereas the total numbers of interneurons were unchanged (Fig. 7C,D; six controls vs six mutants, three sections/animal; $C$, two-way ANOVA, $p$ genotype $0.9999, p$ interaction $=$ 0.0003 ; bin 2 : control mean \pm SEM: $26.6 \% \pm$ 3.64; DKO mean \pm SEM: $16.26 \% \pm 1.60$; Sidak's corrected multiple comparison test, $p<$ $0.0001 ; D$, two-way ANOVA, $p$ genotype $>$ $0.9999, p$ interaction $=0.0016$; str.SP control

\footnotetext{
Values are expressed as the mean \pm SEM $(n=4$ animals per genotype, 3 sections/animal). Statistical differences in str.SP are indicated (Student's $t$ test, $p=0.0164$ ). N.S., Nonstatistical differences in str.IZ/ SVZ (Student's $t$ test, $p=0.803$ ). An assessment of the recombination efficiency of Emx1-Cre is in Extended Data Figure 5-1. Scale bar, $A$, $10 \mu \mathrm{m} ; \boldsymbol{D}, 100 \mu \mathrm{m}$. LV, lateral ventricle.
} 

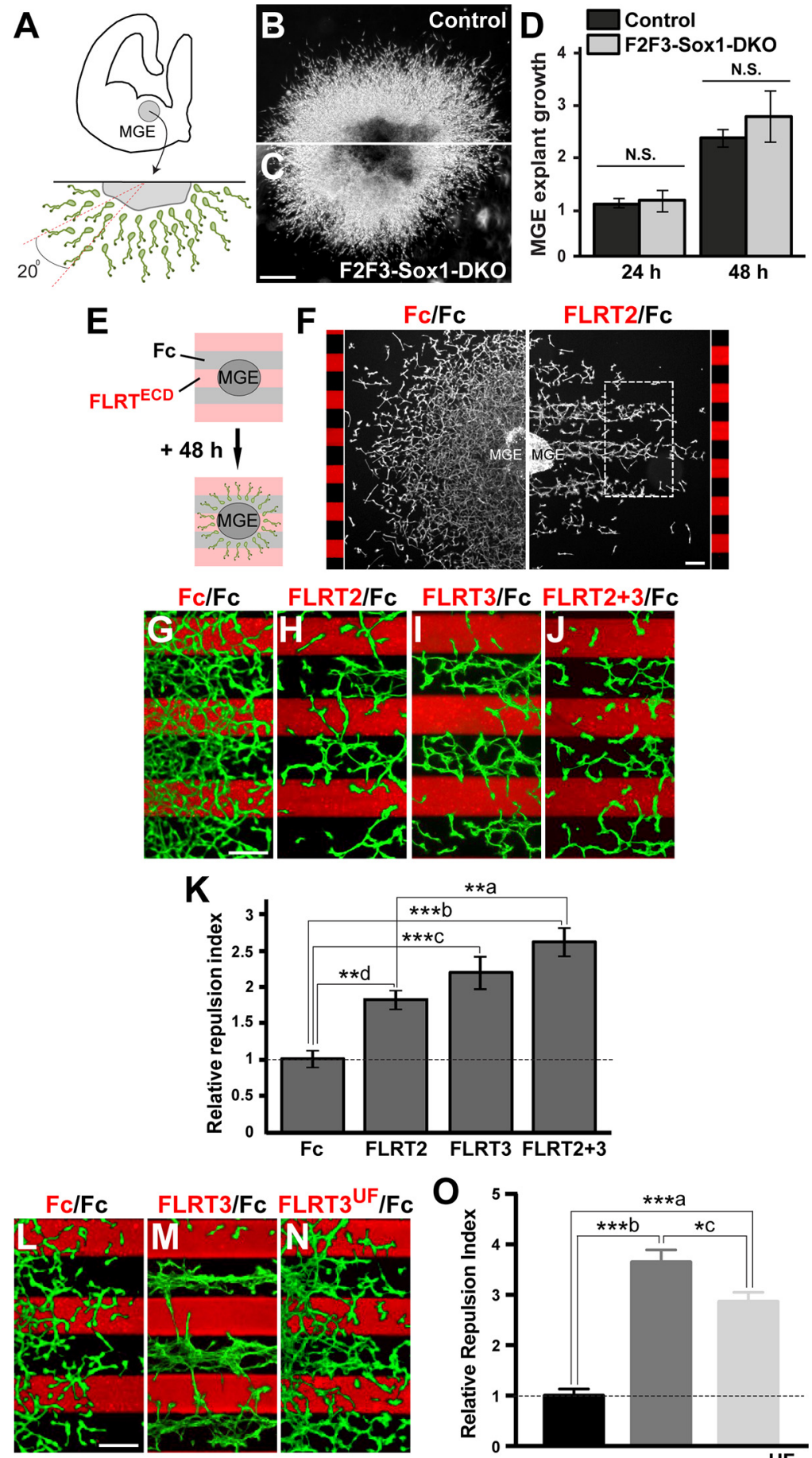

$* * a$

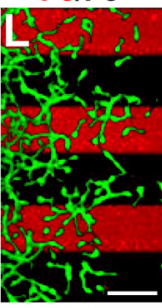

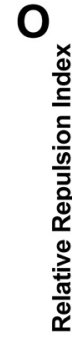

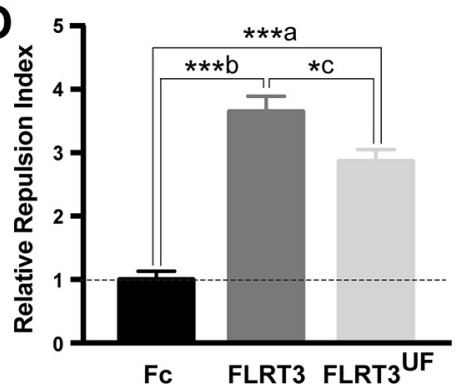

Figure 6. FLRT2 and FLRT3 exert a repulsive effect on MGE-derived interneurons in vitro. A, Diagram of the MGE explant culture from E14.5 embryos to assess the effects of FLRTs in vitro on migrating interneurons (bottom, only half of the explant). Quantification of the growth was assessed by measuring the length from the center of the explant to the most remote interneuron, every $20^{\circ}$ angle. $\boldsymbol{B}, \boldsymbol{C}$, Phase contrast images of representative MGE explants grown for $48 \mathrm{~h}$ in vitro from controls $(\boldsymbol{B})$ or from Sox1-Cre conditional FLRT2; FLRT3 double knock-out embryos (F2F3-Sox1-DKO, C. D, Quantification of the relative MGE explant growth (as indicated in $\boldsymbol{A}$ ) from control (black bars) and from Sox1-Cre conditional FLRT2;FLRT3 double knock-out embryos (F2F3-Sox1-DK0, gray bars), after $24 \mathrm{~h}$ and $48 \mathrm{~h}$ in vitro, as indicated. Values are expressed as the mean fold change to control at $24 \mathrm{~h} \pm \mathrm{SEM}$ ( $n=3-6$ embryos per genotype, 9 measures/explant, 1-6 explants/animal). N.S., Nonstatistical differences (2-way ANOVA; $p$ genotype $=0.1240, p$ interaction $=0.5723$ ). $\boldsymbol{E}$, Stripe assay setup of MGE explants growing on alternate substrates of control protein $(\mathrm{Fc})$ or $\mathrm{FLRT}^{\mathrm{ECD}}-\mathrm{Fc}$ recombinant protein. $\boldsymbol{F}$, Representative images of MGE explants grown for $48 \mathrm{~h}$ on control stripes with alternate Fc proteins (left) or on stripes with alternate $\mathrm{Fc}$ and $\mathrm{FLRT}^{\mathrm{ECD}}-\mathrm{Fc}$ proteins (right). Interneurons were labeled by immunofluorescence with $\beta \mathrm{III}$ tubulin antibodies. Black and red color code indicate the position of the stripes (adjacent to the image) and the recombinant protein mean \pm SEM: $38.48 \% \pm 3.96$, DKO mean \pm SEM: $29.05 \% \pm 2.33$; Sidak's corrected multiple comparison test, $p=0.0064$; data not shown). Notably, this abnormal distribution resembled the one observed in the F2F3-Sox1-DKO or the F2F3Emx-DKO mutants, suggesting that the noncellautonomous effect of FLRTs on interneuron distribution is mediated, at least in part, by Unc5B/D receptors, in vivo.

\section{Discussion}

In the present work we have provided important new insights into the molecular aspects of cortical interneuron development. We report that FLRT proteins are required for the proper formation of the tangential migratory streams of interneurons during cortex development and final laminar allocation. Mechanistically, we provide evidence that FLRTs act noncell autonomously and that this effect is mediated, at least in part, by repulsive signaling of Unc5 receptors.

\section{Molecular mechanisms of FLRT function during interneuron migration}

Migration of interneurons from their place of origin to their final allocation is a multistep process regulated by sequential signaling events that coordinately ensure the proper formation of the inhibitory circuits in the adult. Defects in interneuron migration during development lead to several psychiatric disorders such as autism and schizophrenia (Ross and Walsh, 2001; Marín, 2012), and therefore the study of the molecules regulating this process is fundamental to better understand the etiology of these diseases. FLRT proteins have evolved as key regulators of embryo development in addition to the nervous system, including

used for coating them. G-J, Representative images of MGE-derived interneurons grown on stripes coated with the indicated proteins (black and red color code indicate the position of each stripe and the recombinant protein used for coating them). Interneurons in green were labeled by immunofluorescence with $\beta \mathrm{III}$ tubulin antibodies. $\boldsymbol{K}$, Repulsion index was quantified measuring the percentage of green pixels on the red stripes and applying the formula $y=-0,02 x+1$. Values are expressed as the mean fold change to control $(\mathrm{Fc} / \mathrm{Fc}$ stripe, $\mathrm{Fc}$ control, dashed line) \pm SEM ( $n=40-45$ explants/condition of 4 independent experiments). Statistical differences (1-way ANOVA; $\left.F_{(3,163)}=17.01, p<0.0001\right)$ are indicated, after Tukey's corrected multiple comparison: ${ }^{* * a} p=0.0033,{ }^{* *{ }^{*} p}<0.0001,{ }^{* * c_{p}}<$ $0,0001,{ }^{* * d} p=0,0030$. $\boldsymbol{L}-\boldsymbol{N}$, Representative images of MGE-derived interneurons grown on stripes coated with the indicated proteins, including the Unc5-binding mutant FLRT3 ${ }^{\mathrm{UF}}$ ( $\boldsymbol{N}$; black and red color code indicate the position of each stripe and the recombinant protein used for coating them). Interneurons in green were labeled by immunofluorescence with $\beta \mathrm{III}$ tubulin antibodies. $\boldsymbol{0}$, Repulsion index was quantified measuring the percentage of green pixels on red stripes and applying the formula $y=-0,02 x+1$. Values are expressed as the mean fold change to control ( $\mathrm{Fc} / \mathrm{Fc}$ stripe, $\mathrm{Fc}$ control, dashed line) \pm SEM ( $n=38-45$ explants/condition of 5 independent experiments). Statistical differences (2-way ANOVA; $\mathrm{F}_{(2,121)}=47.17, p<0.0001$ ) are indicated, after Tukey's corrected multiple comparison: ${ }^{* * a} p<0,0001$, ${ }^{* *{ }^{* b}} p<0,0001,{ }^{* \mathrm{c}} p=0,0324$. Scale bar, $\mathbf{C}, \boldsymbol{F}, \mathbf{G}, \mathbf{L}, 100 \mu \mathrm{m}$. 
gastrulation (Egea et al., 2008; Maretto et al., 2008), heart morphogenesis (Müller et al., 2011), and the placenta (Tai-Nagara et al., 2017). FLRTs are unique multifunctional proteins as they act as chemorepellents (Yamagishi et al., 2011; Seiradake et al., 2014), homophilic adhesion molecules (del Toro et al., 2017), coreceptors (Leyva-Díaz et al., 2014), or ternary molecules controlling contact repulsion (del Toro et al., 2020). Up to now, FLRTs have been shown to regulate different aspects of excitatory neuron development including migration, adhesion, axon guidance and synapse formation (Yamagishi et al., 2011; O’Sullivan et al., 2012; Leyva-Díaz et al., 2014; Seiradake et al., 2014; del Toro et al., 2017). In the present work we have demonstrated that FLRT2 and FLRT3 also play an important but redundant role in interneuron development in vivo. This observation is consistent with the expression pattern of FLRTs, specially FLRT3, at these stages and with the fact that both proteins are highly coexpressed in the IZ, where abnormal allocation of interneurons in the FLRT2;FLRT3 double mutants is mainly detected. In the IZ, however, only FLRT3 gene expression was observed by in situ hybridization suggesting that $\mathrm{FLRT}^{2+}$ immunofluorescence in this region could detect the FLRT2 ${ }^{\mathrm{ECD}}$ cleaved and released from the adjacent CP (Yamagishi et al., 2011) or the expression of FLRT2 by axons (corticofugal or thalamocortical) en passant. Redundancy of FLRT proteins was previously suggested. For instance, the three mammalian FLRT proteins display identical structure with high homology ( $>40 \%$ in humans) and similarity ( $>15 \%$ in humans; Lacy et al., 1999). Moreover, FLRT2 and FLRT3 are interchangeable and sufficient to rescue functional activities in the anterior visceral endoderm and the developing epicardium of the mouse embryo (Müller et al., 2011). Finally, the double ablation of FLRT1;FLRT3 causes defects in adhesion and migration speed of radial migrating progenitors that leads to spontaneous sulcus formation in the mouse cortex (del Toro et al., 2017).

Our genetic experiments indicate that FLRTs have a noncell autonomous effect of cortical interneurons. To date, the molecules controlling interneuron distribution within the developing cortex showed a chemoattractant effect in MGE-derived interneurons in vitro, including SDF/Cxcl12 and neuregulins (Yau et al., 2003; Flames et al., 2004; Li et al., 2008; López-Bendito et al., 2008). However, using similar in vitro MGE explant assays, we showed that FLRTs exerted a repulsive effect on interneurons similar to that described for excitatory neurons and endothelial cells (Yamagishi et al., 2011; Seiradake et al., 2014). This effect is compatible with the abnormal distribution of interneurons observed in the IZ of the F2F3-Sox1-DKOs and strongly suggests that FLRT2 and FLRT3 in the IZ help to keep interneuron stream organization by acting as repulsive cues. Interestingly, our quantitative analysis indicates that the majority of interneurons abnormally allocated in the IZ of the F2F3-Sox1-DKOs arises from the SP stream, suggesting that FLRT2 and FLRT3 are specially required for the maintenance of this particular stream.

It has been proposed that the restricted formation of the MZ and the IZ/SVZ streams results from the strong expression of the chemokine SDF1/Cxcl12 by the meninges and by Intermediate Progenitor Cells in the SVZ, respectively, by its potent chemoattractant activity for interneurons in vitro together with its limited diffusion in vivo (Tham et al., 2001; Stumm et al., 2003; Tiveron et al., 2006; Li et al., 2008; López-Bendito et al., 2008; SánchezAlcañiz et al., 2011). It is assumed that the establishment of the SP stream is also controlled by SDF/Cxcl12, but the expression of this chemokine in the region where the SP stream proceeds is much lower (Stumm et al., 2007). It is conceivable therefore that in this case these low levels are not sufficient to keep interneurons confined in the SP stream and that additional mechanisms are involved. According to our results, we propose that FLRT2 and FLRT3 could exert a counterbalance repulsive force in the IZ to keep SP stream organization.

We have previously shown that FLRT-Unc5 repulsive response controls the correct radial migration of excitatory neuron progenitors in the developing cortex (Yamagishi et al., 2011). By using mutant recombinant FLRT protein unable to bind Unc5 receptors in stripe assays, we could demonstrate that Unc5s also mediate the repulsive effects of FLRTs on interneurons. Nevertheless, repulsion was not completely abolished, indicating that other mechanisms are involved. Recently, FLRT binding to the synaptogenic protein complex latrophilinsteneurins has been shown to direct the migration of neurons by a contact repulsion-dependent mechanism (del Toro et al., 2020). Further investigation will be required to address the implication of this ternary complex in interneuron migration. Alternatively, FLRTs have been shown to be able to finely regulate adhesion (through homotypic binding) and repulsion (through heterotypic binding to Unc5 receptors) simultaneously (Seiradake et al., 2014). It is therefore 
possible that the disruption of this adhesion/repulsion equilibrium might affect interneuron development. In any case, the participation of Unc5 receptors in cortical interneuron development seems to be relevant in vivo as the double Unc5B;Unc5D mutants displayed a significant change in the distribution of $\mathrm{CB}+$ interneurons. Importantly, this change affected in particular SP stream interneurons, very similar to what we observed in the double FLRT2;FLRT3 knock-out animals, which reinforces the idea that Unc5s mediate the chemorepulsive effect of FLRTs for the maintenance of the cortical interneuron streams in vivo.

\section{Specific mechanisms of interneuron distribution during cortex development}

The proper formation of the three intracortical streams of migrating interneurons is essential for the timing of invasion of the $\mathrm{CP}$ and the correct development of cortical inhibitory circuits (López-Bendito et al., 2008). Despite cortical interneurons at this stage showing a relatively uniform appearance (Martini et al., 2009), recent data suggests an early interneuron specification, occurring already in the SVZ/VZ soon after interneuron progenitors become postmitotic, that determines the route of migration in the cortex (Mi et al., 2018). For instance, a particular suptype of SST + interneurons, the Martinotti cells, as well as the translaminar PV + interneurons show a strong preference for migration through the MZ stream (Lim et al., 2018). In accordance with this model, several transcriptomic approaches have demonstrated that interneurons from different streams display different gene expression, including, interestingly, FLRT2 and FLRT3, which would allow these cells to sort out a specific migration route (Antypa et al., 2011; Lim et al., 2018). So far, however, evidence for specific mechanisms that could regulate the migration of interneurons through a particular stream is missing. Our observation that the distribution of the $\mathrm{CB}+$ interneurons is specifically affected in the SP region in the double mutants FLRT2; FLRT3 is particularly compelling and suggest that FLRT proteins have a specific function in the confinement of interneurons in the SP stream.

In the adult cortex of the double mutants FLRT2;FLRT3, the laminar distribution of SST + interneurons is abnormal with a higher percentage of SST+ interneurons in layer 6 and less in layer 5 (both layers containing Martinotti and non-Martinotti interneurons; Yavorska and Wehr, 2016). In mice, a small fraction $(\sim 7,5 \%)$ of SST + interneurons in the somatosensory cortex do coexpress CR in layers 5 and 6 (Xu et al., 2006), probably too few to detect changes in the whole $\mathrm{CR}+$ interneuron counting analysis. Alternatively it could be that in the double FLRT2; FLRT3 mutants, only the STT + ;CR- interneurons in layers 5 and 6 are affected. In any case, considering the developmental defect concerning the SP stream in the mutants, it is tempting to speculate that at least some SP interneurons would finally be allocated in layers 5 and 6 giving rise to SST + interneurons, in particular non-Martinotti cells (Lim et al., 2018). In the future it would be extremely valuable to generate appropriate genetic tools based on specific stream gene expression to perform fatemapping studies and follow the final destination of interneurons of a particular stream.

In summary, in this study we have expanded the spectrum of functions of the multifunctional FLRT proteins and demonstrated the important role of FLRTs for interneuron development during the formation of the tangential migratory streams in the cortex and for laminar allocation in vivo.

\section{References}

Anderson SA, Eisenstat DD, Shi L, Rubenstein JL (1997) Interneuron migration from basal forebrain to neocortex: dependence on Dlx genes. Science 278:474-476.

Ang ESBC, Haydar TF, Gluncic V, Rakic P (2003) Four-dimensional migratory coordinates of GABAergic interneurons in the developing mouse cortex. J Neurosci 23:5805-5815.

Antypa M, Faux C, Eichele G, Parnavelas JG, Andrews WD (2011) Differential gene expression in migratory streams of cortical interneurons. Eur J Neurosci 34:1584-1594.

Bartolini G, Sánchez-Alcañiz JA, Osório C, Valiente M, García-Frigola C, Marín O (2017) Neuregulin 3 mediates cortical plate invasion and laminar allocation of GABAergic interneurons. Cell Rep 18:1157-1170.

Chan CH, Godinho LN, Thomaidou D, Tan SS, Gulisano M, Parnavelas JG (2001) Emxl is a marker for pyramidal neurons of the cerebral cortex. Cereb Cortex 11:1191-1198.

del Toro D, Ruff T, Cederfäll E, Villalba A, Seyit-Bremer G, Borrell V, Klein R (2017) Regulation of cerebral cortex folding by controlling neuronal migration via FLRT adhesion molecules. Cell 169:621-635. e16.

del Toro D, Carrasquero-Ordaz MA, Chu A, Ruff T, Shahin M, Jackson VA, Chavent M, Berbeira-Santana M, Seyit-Bremer G, Brignani S, Kaufmann R, Lowe E, Klein R, Seiradake E (2020) Structural basis of teneurin-latrophilin interaction in repulsive guidance of migrating neurons. Cell 180:323-339.

Egea J, Nissen UV, Dufour A, Sahin M, Greer P, Kullander K, Mrsic-Flogel TD, Greenberg ME, Kiehn O, Vanderhaeghen P, Klein R (2005) Regulation of EphA 4 kinase activity is required for a subset of axon guidance decisions suggesting a key role for receptor clustering in Eph function. Neuron 47:515-528.

Egea J, Erlacher C, Montanez E, Burtscher I, Yamagishi S, Hess M, Hampel F, Sanchez R, Rodriguez-Manzaneque MT, Bösl MR, Fässler R, Lickert H, Klein R (2008) Genetic ablation of FLRT3 reveals a novel morphogenetic function for the anterior visceral endoderm in suppressing mesoderm differentiation. Genes Dev 22:3349-3362.

Evsyukova I, Plestant C, Anton ES (2013) Integrative mechanisms of oriented neuronal migration in the developing brain. Annu Rev Cell Dev Biol 29:299-353.

Faux C, Rakic S, Andrews W, Britto JM (2012) Neurons on the move: migration and lamination of cortical interneurons. Neurosignals 20:168-189.

Flames N, Long JE, Garratt AN, Fischer TM, Gassmann M, Birchmeier C, Lai C, Rubenstein JLR, Mar1 O, Francisco S (2004) Short- and long-range attraction of cortical GABAergic interneurons by neuregulin-1. Neuron 44:251-261.

Franklin K, Paxinos G (2008) The mouse brain in strereotaxic coordinates. San Diego, CA: Academic Press.

Gonchar Y, Burkhalter A (1997) Three distinct families of GABAergic neurons in rat visual cortex. Cereb Cortex 7:347-358.

Gorski JA, Talley T, Qiu M, Puelles L, Rubenstein JLR, Jones KR (2002) Cortical excitatory neurons and glia, but not GABAergic neurons, are produced in the Emx1-expressing lineage. J Neurosci 22:6309-6314.

Hernández-Miranda LR, Parnavelas JG, Chiara F (2010) Molecules and mechanisms involved in the generation and migration of cortical interneurons. ASN Neuro 2:e00031.

Kandel ER, Schwartz JH, Jessell T (2000) Principles of neural science. New York, NY: McGraw-Hill.

Lacy SE, Bönnemann CG, Buzney EA, Kunkel LM (1999) Identification of FLRT1, FLRT2, and FLRT3 : a novel family of transmembrane leucinerich repeat proteins. Genomics 62:417-426.

Lamprecht MR, Sabatini DM, Carpenter AE (2007) CellProfiler: free, versatile software for automated biological image analysis. Biotechniques 42:71-75.

Leyva-Díaz E, del Toro D, Menal MJ, Cambray S, Susín R, Tessier-Lavigne M, Klein R, Egea J, López-Bendito G (2014) FLRT3 is a Robo1-interacting protein that determines Netrin- 1 attraction in developing axons. Curr Biol 24:494-508.

Li G, Adesnik H, Li J, Long J, Nicoll RA, Rubenstein JLR, Pleasure SJ (2008) Regional distribution of cortical interneurons and development of inhibitory tone are regulated by Cxcl12/Cxcr4 signaling. J Neurosci 28:10851098.

Lim L, Pakan JMP, Selten MM, Marques-Smith A, Llorca A, Bae SE, Rochefort NL, Marín O (2018) Optimization of interneuron function by 
direct coupling of cell migration and axonal targeting. Nat Neurosci 21:920-931.

Lodato S, Rouaux C, Quast KB, Jantrachotechatchawan C, Studer M, Hensch TK, Arlotta P (2011) Excitatory projection neuron subtypes control the distribution of local inhibitory interneurons in the cerebral cortex. Neuron 69:763-779.

López-Bendito G, Sánchez-Alcañiz JA, Pla R, Borrell V, Picó E, Valdeolmillos M, Marín O (2008) Chemokine signaling controls intracortical migration and final distribution of GABAergic interneurons. J Neurosci 28:1613-1624.

Lu X, Le Noble F, Yuan L, Jiang Q, De Lafarge B, Sugiyama D, Bréant C, Claes F, De Smet F, Thomas JL, Autiero M, Carmeliet P, Tessier-Lavigne M, Eichmann A (2004) The netrin receptor UNC5B mediates guidance events controlling morphogenesis of the vascular system. Nature 432:179-186.

Maretto S, Müller PS, Aricescu AR, Cho KW, Bikoff EK, Robertson EJ (2008) Ventral closure, headfold fusion and definitive endoderm migration defects in mouse embryos lacking the fibronectin leucine-rich transmembrane protein FLRT3. Dev Biol 318:184-193.

Marín O (2012) Interneuron dysfunction in psychiatric disorders. Nat Rev Neurosci 13:107-120.

Martini FJ, Valiente M, López Bendito G, Szabó G, Moya F, Valdeolmillos M, Marín O (2009) Biased selection of leading process branches mediates chemotaxis during tangential neuronal migration. Development 136:4150.

Mi D, Li Z, Lim L, Li M, Moissidis M, Yang Y, Gao T, Hu TX, Pratt T, Price DJ, Sestan N, Marín O (2018) Early emergence of cortical interneuron diversity in the mouse embryo. Science 360:81-85.

Moore SW, Tessier-Lavigne M, Kennedy TE (2007) Netrins and their receptors. Adv Exp Med Biol 621:17-31.

Müller P-S, Schulz R, Maretto S, Costello I, Srinivas S, Bikoff E, Robertson E (2011) The fibronectin leucine-rich repeat transmembrane protein Flrt2 is required in the epicardium to promote heart morphogenesis. Development 138:1297-1308.

Nadarajah B, Alifragis P, Wong ROL, Parnavelas JG (2002) Ventricledirected migration in the developing cerebral cortex. Nat Neurosci 5:218-224.

Nery S, Fishell G, Corbin JG (2002) The caudal ganglionic eminence is a source of distinct cortical and subcortical cell populations. Nat Neurosci 5:1279-1287.

O'Sullivan ML, de Wit J, Savas JN, Comoletti D, Otto-Hitt S, Yates JR, Ghosh A (2012) FLRT proteins are endogenous latrophilin ligands and regulate excitatory synapse development. Neuron 73:903-910.

Paixão S, Balijepalli A, Serradj N, Niu J, Luo W, Martin JH, Klein R (2013) EphrinB3/EphA4-mediated guidance of ascending and descending spinal tracts. Neuron 80:1407-1420.

Peyre E, Silva CG, Nguyen L (2015) Crosstalk between intracellular and extracellular signals regulating interneuron production, migration and integration into the cortex. Front Cell Neurosci 9:129.

Pfeffer CK, Xue M, He M, Huang ZJ, Scanziani M (2013) Inhibition of inhibition in visual cortex: the logic of connections between molecularly distinct interneurons. Nat Neurosci 16:1068-1076.

Ross ME, Walsh CA (2001) Human brain malformations and their lessons for neuronal migration. Annu Rev Neurosci 24:1041-1070.

Rudy B, Fishell G, Lee S, Hjerling-Leffler J (2011) Three groups of interneurons account for nearly $100 \%$ of neocortical GABAergic neurons. Dev Neurobiol 71:45-61.

Sánchez-Alcañiz JA, Haege S, Mueller W, Pla R, Mackay F, Schulz S, LópezBendito G, Stumm R, Marín O (2011) Cxcr7 controls neuronal migration by regulating chemokine responsiveness. Neuron 69:77-90.

Schambra UB (2009). Prenatal mouse brain atlas. New York, NY, Springer.

Seiradake E, del Toro D, Nagel D, Cop F, Härtl R, Ruff T, Seyit-Bremer G, Harlos K, Border EC, Acker-Palmer A, Jones EY, Klein R (2014) FLRT structure: balancing repulsion and cell adhesion in cortical and vascular development. Neuron 84:370-385.
Stumm R, Kolodziej A, Schulz S, Kohtz JD, Höllt V (2007) Patterns of SDF1alpha and SDF-1gamma mRNAs, migration pathways, and phenotypes of CXCR4-expressing neurons in the developing rat telencephalon. J Comp Neurol 502:382-399.

Stumm RK, Zhou C, Ara T, Lazarini F, Dubois-Dalcq M, Nagasawa T, Höllt V, Schulz S (2003) CXCR4 regulates interneuron migration in the developing neocortex. J Neurosci 23:5123-5130.

Tai-Nagara I, Yoshikawa Y, Numata N, Ando T, Okabe K, Sugiura Y, Ieda M, Takakura N, Nakagawa O, Zhou B, Okabayashi K, Suematsu M, Kitagawa Y, Bastmeyer M, Sato K, Klein R, Navankasattusas S, Li DY, Yamagishi S, Kubota Y (2017) Placental labyrinth formation in mice requires endothelial FLRT2/UNC5B signaling. Development 144:23922401.

Takashima Y, Era T, Nakao K, Kondo S, Kasuga M, Smith AG, Nishikawa SI (2007) Neuroepithelial cells supply an initial transient wave of MSC differentiation. Cell 129:1377-1388.

Tanaka D, Nakaya Y, Yanagawa Y, Obata K, Murakami F (2003) Multimodal tangential migration of neocortical GABAergic neurons independent of GPI-anchored proteins. Development 130:5803-5813.

Tanaka DH, Nakajima K (2012) Migratory pathways of GABAergic interneurons when they enter the neocortex. Eur J Neurosci 35:1655-1660.

Tham TN, Lazarini F, Franceschini IA, Lachapelle F, Amara A, DuboisDalcq M (2001) Developmental pattern of expression of the alpha chemokine stromal cell-derived factor 1 in the rat central nervous system. Eur J Neurosci 13:845-856.

Tiveron M-C, Rossel M, Moepps B, Zhang YL, Seidenfaden R, Favor J, König $\mathrm{N}$, Cremer H (2006) Molecular interaction between projection neuron precursors and invading interneurons via stromal-derived factor 1 (CXCL12)/CXCR4 signaling in the cortical subventricular zone/intermediate zone. J Neurosci 26:13273-13278.

Tremblay R, Lee S, Rudy B (2016) GABAergic interneurons in the neocortex: from cellular properties to circuits. Neuron 91:260-292.

van den Berghe V, Stappers E, Vandesande B, Dimidschstein J, Kroes R, Francis A, Conidi A, Lesage F, Dries R, Cazzola S, Berx G, Kessaris N, Vanderhaeghen P, van Ijcken W, Grosveld FG, Goossens S, Haigh JJ, Fishell G, Goffinet A, Aerts S, et al. (2013) Directed migration of cortical interneurons depends on the cell-autonomous action of Sip1. Neuron 77:70-82.

Wang Y, Li G, Stanco A, Long JE, Crawford D, Potter GB, Pleasure SJ, Behrens T, Rubenstein JLR (2011) CXCR4 and CXCR7 have distinct functions in regulating interneuron migration. Neuron 69:61-76.

Wichterle H, Garcia-Verdugo JM, Herrera DG, Alvarez-Buylla A (1999) Young neurons from medial ganglionic eminence disperse in adult and embryonic brain. Nat Neurosci 2:461-466.

Wilson BD, Ii M, Park KW, Suli A, Sorensen LK, Larrieu-Lahargue F, Urness LD, Suh W, Asai J, Kock GA, Thorne T, Silver M, Thomas KR, Chien CB, Losordo DW, Li DY (2006) Netrins promote developmental and therapeutic angiogenesis. Science 313:640-644.

Xu X, Roby KD, Callaway EM (2006) Mouse cortical inhibitory neuron type that coexpresses somatostatin and calretinin. J Comp Neurol 499:144160.

Yamagishi S, Hampel F, Hata K, Del Toro D, Schwark M, Kvachnina E, Bastmeyer M, Yamashita T, Tarabykin V, Klein R, Egea J (2011) FLRT2 and FLRT3 act as repulsive guidance cues for Unc5-positive neurons. EMBO J 30:2920-2933.

Yau H-J, Wang H-F, Lai C, Liu F-C (2003) Neural development of the neuregulin receptor ErbB4 in the cerebral cortex and the hippocampus: preferential expression by interneurons tangentially migrating from the ganglionic eminences. Cereb Cortex 13:252-264.

Yavorska I, Wehr M (2016) Somatostatin-expressing inhibitory interneurons in cortical circuits. Front Neural Circuits 10:1-18.

Zarbalis K, Choe Y, Siegenthaler JA, Orosco LA, Pleasure SJ (2012) Meningeal defects alter the tangential migration of cortical interneurons in Foxclhith/hith mice. Neural Dev 7:2. 